\title{
NATURALITY PROPERTIES AND COMPARISON RESULTS FOR TOPOLOGICAL AND INFINITESIMAL EMBEDDED JUMP LOCI
}

\author{
STEFAN PAPADIMA ${ }^{1} \uparrow$ AND ALEXANDER I. SUCIU ${ }^{2}$
}

\begin{abstract}
AвSTRACT. We use augmented commutative differential graded algebra (ACDGA) models to study $G$-representation varieties of fundamental groups $\pi=\pi_{1}(M)$ and their embedded cohomology jump loci, around the trivial representation 1 . When the space $M$ admits a finite family of maps, uniformly modeled by ACDGA morphisms, and certain finiteness and connectivity assumptions are satisfied, the germs at 1 of $\operatorname{Hom}(\pi, G)$ and of the embedded jump loci can be described in terms of their infinitesimal counterparts, naturally with respect to the given families. This approach leads to fairly explicit answers when $M$ is either a compact Kähler manifold, the complement of a central complex hyperplane arrangement, or the total space of a principal bundle with formal base space, provided the Lie algebra of the linear algebraic group $G$ is a non-abelian subalgebra of $\mathfrak{s}_{2}(\mathbb{C})$.
\end{abstract}

\section{Contents}

1. Introduction and statement of results 2

2. Artin approximation 7

3. Algebraic models of spaces and maps 8

4. Deformation theory of representation varieties 13

5. Cohomology jump loci and naturality properties 17

6. A natural comparison between embedded jump loci 23

7. Kähler manifolds 28

8. Principal bundles 33

9. Quasi-projective manifolds $\quad 37$

Acknowledgement 43

References 43

2010 Mathematics Subject Classification. Primary 14B12, 14F35, 55N25, 55P62. Secondary 20C15, $57 \mathrm{~S} 15$.

Key words and phrases. Representation variety, flat connection, cohomology jump loci, filtered differential graded algebra, relative minimal model, mixed Hodge structure, analytic local ring, Artinian local ring, differential graded Lie algebra, deformation theory, formal spaces and maps, quasi-compact Kähler manifold, hyperplane arrangement, principal bundle.

${ }^{1}$ Work partially supported by the Romanian Ministry of Research and Innovation, CNCS-UEFISCDI, grant PN-III-P4-ID-PCE-2016-0030, within PNCDI III.

$\dagger$ Deceased January 10, 2018.

${ }^{2}$ Partially supported by the Simons Foundation collaboration grant for mathematicians 354156. 


\section{INTRODUCTION AND STATEMENT OF RESULTS}

1.1. Representation varieties and jump loci. Sheaf cohomology is ubiquitous in geometry and topology. The parameter space for rank $n$ locally constant sheaves on a pathconnected, pointed CW-complex $X$ with finitely many 1-cells may be identified with the $\mathrm{GL}_{n}$-representation variety of the fundamental group of $X$. Twisted cohomology on $X$ is encoded by the filtrations of these varieties by the (embedded) jump loci. While $\mathrm{GL}_{1}$ representation varieties are finite unions of affine tori, the picture changes dramatically in higher rank. For instance, the universality theorem of Kapovich and Millson [18] states that $\mathrm{PSL}_{2}$-representation varieties may have arbitrarily bad singularities, away from the origin 1 (the trivial representation). This is the reason why we focus here on analytic germs at the origin of the embedded cohomology jump loci. The general case is analyzed by Budur and Wang in [4], but it seems that explicit computations away from 1 are intractable in full generality.

By the main result from [9], Theorem B, the germs at 1 of the embedded jump loci of $X$ are isomorphic to the germs at 0 of the infinitesimal embedded jump loci of a commutative, differential graded algebra $A$, provided this CDGA models $X$, and certain mild finiteness assumptions are satisfied. Furthermore, in the abelian case, this identification is natural. One of our main goals here is to extend this natural comparison to the nonabelian setting, by studying the behavior of jump loci under suitable continuous maps between spaces and CDGA maps between their models.

By construction, both representation varieties and their infinitesimal analogues are (bi)functorial. The naturality properties of both types of jump loci are summarized in Corollaries 5.8 and 5.10. As we point out in Example 5.9, naturality at this level requires connectivity assumptions for maps, defined in $\S 3.1$. What greatly simplifies things in the abelian case is the existence of a global, exponential map which relates representation varieties to their infinitesimal counterparts. By way of contrast, it follows from Example 7.10 that no such map exists in the $\mathrm{PSL}_{2}$ case, even for compact Riemann surfaces.

To avoid this major difficulty, we construct local analytic isomorphisms between the two types of jump loci by means of Artin approximation. That is, we replace the respective local analytic rings by their completions (or by functors of Artin rings), and deduce local analytic naturality from naturality at the level of completions. This we do in Proposition 2.3, which is a general result about simultaneous Artin approximation. We need this 'simultaneous' framework in view of the applications to be derived later on, which involve families of maps between spaces.

The condition that makes our approach to a natural comparison between embedded jump loci work is based on the $q$-equivalence relation for morphisms between augmented commutative differential graded algebras (ACDGAs), denoted by $\simeq_{q}$, and detailed in Definition 3.2. The primary examples we have in mind are the ACDGA morphisms $\Omega(f)$ between Sullivan-de Rham models induced by pointed, continuous maps between topological spaces. 
1.2. Natural comparison with respect to finite families of maps. In more detail, the embedded jump loci we consider in this paper are as follows. First let $X$ be a pointed, pathconnected space with fundamental group $\pi$, and let $\iota: G \rightarrow \mathrm{GL}(V)$ be a representation. For each $i, r \geqslant 0$, the embedded jump locus of $X$ with respect to $\iota$ is the pair

$$
\left(\operatorname{Hom}(\pi, G), \mathscr{V}_{r}^{i}(X, \iota)\right),
$$

where $\mathscr{V}_{r}^{i}(X, \iota)$ is the set of homomorphisms $\rho$ for which the $i$-th cohomology group of $X$ with coefficients in the local system $V_{\iota \circ \rho}$ has dimension at least $r$.

Next, let $A$ be a CDGA, and let $\theta: \mathfrak{g} \rightarrow \mathfrak{g l}(V)$ be a Lie algebra representation. The infinitesimal analog of the representation variety is the set $\mathscr{F}(A, \mathfrak{g}) \subseteq A^{1} \otimes \mathfrak{g}$ of $\mathfrak{g}$-valued flat connections on $A$. For each $i, r \geqslant 0$, the infinitesimal embedded jump locus of $A$ with respect to $\theta$ is the pair

$$
\left(\mathscr{F}(A, \mathfrak{g}), \mathscr{R}_{r}^{i}(A, \theta)\right),
$$

where $\mathscr{R}_{r}^{i}(A, \theta)$ is the set of flat connections $\omega$ for which the $i$-th cohomology group of the cochain complex $\left(A \otimes V, d_{\omega}\right)$ defined in (5.2) has dimension at least $r$.

Assume now that $\iota$ is a rational representation of linear algebraic groups, and both $\mathfrak{g}$ and $V$ are finite-dimensional. Under mild $q$-finiteness conditions on $X$ and $A$ (explained in $\S 3.2)$, both $\operatorname{Hom}(\pi, G)$ and $\mathscr{F}(A, \mathfrak{g})$ are affine varieties, and their jump loci are closed subvarieties, for all $i \leqslant q$ and $r \geqslant 0$.

We may now state our first main result. Let $\left\{f: X \rightarrow X_{f}\right\}_{f \in E}$ be a finite family of continuous maps between pointed, path-connected spaces. For each $f \in E$, we denote by $f_{\sharp}: \pi \rightarrow \pi_{f}$ the induced homomorphism on fundamental groups. Let also $\left\{\Phi_{f}: A_{f} \rightarrow\right.$ $A\}_{f \in E}$ be a family of ACDGA morphisms. Consider a rational representation of linear algebraic groups, $\iota: G \rightarrow \mathrm{GL}(V)$, over $\mathbb{k}=\mathbb{R}$ or $\mathbb{C}$, with tangential representation $\theta: \mathfrak{g} \rightarrow$ $\mathfrak{g l}(V)$. For an affine $\mathbb{k}$-variety $\mathscr{X}$, we denote by $\mathscr{X}_{(x)}$ the $\mathbb{k}$-analytic germ of $\mathscr{X}$ at a point $x \in \mathscr{X}$.

Theorem 1.1. Fix an integer $q \geqslant 1$, and suppose the following conditions hold:

(1) All the above spaces and CDGAs are q-finite.

(2) Both $f$ and $\Phi_{f}$ are $(q-1)$-connected maps, for all $f \in E$.

(3) $\Omega(f) \simeq_{q} \Phi_{f}$ in ACDGA, uniformly with respect to $f \in E$.

Under these assumptions, we may find local analytic isomorphisms $a: \mathscr{F}(A, \mathfrak{g})_{(0)} \stackrel{\simeq}{\longrightarrow}$ $\operatorname{Hom}(\pi, G)_{(1)}$ and $a_{f}: \mathscr{F}\left(A_{f}, \mathfrak{g}\right)_{(0)} \stackrel{\simeq}{\longrightarrow} \operatorname{Hom}\left(\pi_{f}, G\right)_{(1)}$ for all $f \in E$ with the property that the following diagram commutes, for all $f \in E$ :

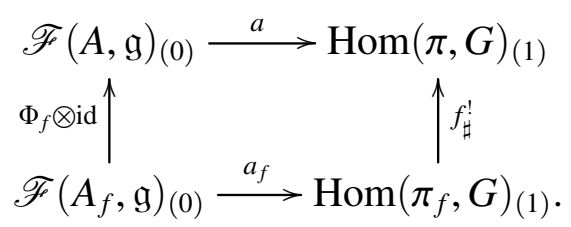


Moreover, this construction induces the following commuting diagram of (local, reduced) embedded jump loci, for all $f \in E, i \leqslant q$, and $r \geqslant 0$ :

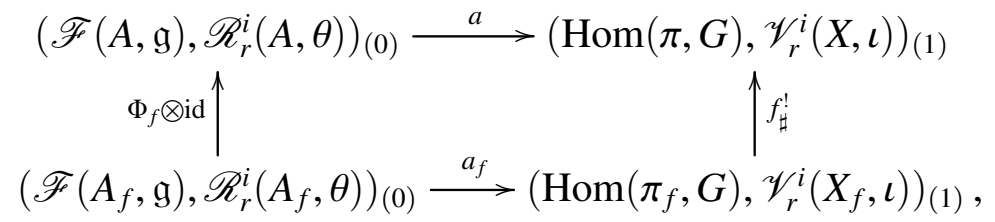

where both horizontal arrows are isomorphisms of analytic pairs.

The meaning of the $q$-equivalence relation between continuous pointed maps and ACDGA maps, uniformly with respect to finite families, is explained in Definition 6.3. For a oneelement family $\{f\}$, this condition simply means that $\Omega(f) \simeq_{q} \Phi_{f}$ in ACDGA. For a certain type of two-element family, the uniformity condition is verified in the next theorem.

Let $f: X \rightarrow Y$ be a continuous, pointed map between path-connected spaces. Let $\pi$ be the fundamental group of $X$, let abf : $\pi \rightarrow \pi_{\text {abf }}$ be the projection onto its maximal torsionfree abelian quotient, and let $f_{0}: X \rightarrow K\left(\pi_{\mathrm{abf}}, 1\right)$ be a classifying map for this projection. Set $A_{0}^{\bullet}=\left(\bigwedge^{\bullet} H^{1}(X), d=0\right)$.

Theorem 1.2. Suppose that $X$ and $Y$ are $q$-finite, for some $q \geqslant 1$, and $\Omega(f) \simeq_{q} \Phi$ in ACDGA, where $\Phi: A_{Y} \rightarrow A_{X}$ is a morphism between q-finite ACDGAs. There is then an ACDGA map $\Phi_{0}: A_{0} \rightarrow A_{X}$ inducing an isomorphism on $H^{1}$, and such that $\Omega\left(f_{0}\right) \simeq_{q} \Phi_{0}$ in ACDGA, uniformly with respect to the families $\left\{f, f_{0}\right\}$ and $\left\{\Phi, \Phi_{0}\right\}$. Moreover, if $f$ and $\Phi$ are 0-connected maps, then all hypotheses from Theorem 1.1 are satisfied for $q=1$.

1.3. A general framework for applications. Let $\pi$ be the fundamental group of a 1finite manifold $M$. We aim at finding structural results for (non-abelian) embedded jump loci of $M$ near the origin, in low degrees. To start with, we want to extract from the geometry of $M$ a finite family of group epimorphisms, $\left\{f_{\sharp}: \pi \rightarrow \pi_{f}\right\}_{f \in \mathscr{E}(M)}$, induced on fundamental groups by maps $f: M \rightarrow M_{f}$ onto manifolds of smaller dimension. Next, we set $E(M)=\mathscr{E}(M) \cup\left\{f_{0}\right\}$, where $f_{0}$ is a classifying map for the projection abf : $\pi \rightarrow \pi_{\text {abf }}$.

Let $\iota: G \rightarrow \mathrm{GL}(V)$ be a rational representation of $\mathbb{C}$-linear algebraic groups. For a group homomorphism $h: \pi \rightarrow \pi^{\prime}$, we let $h^{!}: \operatorname{Hom}\left(\pi^{\prime}, G\right) \rightarrow \operatorname{Hom}(\pi, G)$ denote the induced morphism between the corresponding representation varieties. As explained in Remark 7.8, the abelian part of $\operatorname{Hom}(\pi, G)$ near the trivial representation coincides with the germ $\operatorname{abf}^{!}\left(\operatorname{Hom}\left(\pi_{\mathrm{abf}}, G\right)\right)_{(1)}$. By naturality of jump loci, the natural inclusion,

$$
\operatorname{Hom}(\pi, G) \supseteq \bigcup_{f \in E(M)} f_{\sharp}^{!} \operatorname{Hom}\left(\pi_{f}, G\right),
$$

induces inclusions

$$
\mathscr{V}_{r}^{i}(M, \iota) \supseteq \bigcup_{f \in E(M)} f^{!} \mathscr{V}_{r}^{i}\left(M_{f}, \iota\right)
$$


for all $i \leqslant 1$ and $r \geqslant 1$. Finally, we ask whether the inclusions (1.3) and (1.4) become equalities near 1 .

We focus on the rank 2 case, when the Lie algebra $g$ of $G$ is a non-abelian subalgebra of $\mathfrak{s l}_{2}(\mathbb{C})$. Our techniques allow us to treat simultaneously two interesting classes of examples: (1) quasi-compact Kähler manifolds (in particular, quasi-projective manifolds), and (2) closed, smooth manifolds endowed with a free action of a compact, connected, real Lie group $K$. In the first case, the family $\mathscr{E}(M)$ consists of equivalence classes of 'admissible' maps (in the sense of Arapura [2]) from $M$ to smooth complex curves of negative Euler characteristic. In the second case, $\mathscr{E}(M)$ has only one element, namely the bundle projection $M \rightarrow M / K$.

When the group $G$ is $\mathrm{SL}_{2}(\mathbb{C})$ or $\mathrm{PSL}_{2}(\mathbb{C})$ and $M$ is a quasi-projective manifold, equality in (1.3) is related to deep results of Corlette-Simpson [6] and Loray-Pereira-Touzet [20], which give a rather intricate classification for the $G$-representations of $\pi_{1}(M)$, also valid away from 1 . When $M$ is a quasi-compact Kähler manifold, $\iota=\operatorname{id}_{\mathbb{C}^{\times}}$, and $i=r=1$, equality in (1.4) near 1 is equivalent to the subtle description of $\mathscr{V}_{1}^{1}(M, \iota)$ from [2], again also valid away from 1 . Thus, our results below may be viewed as a more precise version of the aforementioned work, in a broader context, albeit only near the origin.

Theorem 1.3. Let $G$ be a $\mathbb{C}$-linear algebraic group with non-abelian Lie algebra $\mathfrak{g} \subseteq$ $\mathfrak{s l}_{2}(\mathbb{C})$, and let $\iota: G \rightarrow \mathrm{GL}(V)$ be a rational representation. For $i=r=1$ and for $i=0$, $r \geqslant 1$, both (1.3) and (1.4) become equalities near the origin 1, provided $\pi=\pi_{1}(M)$ and either

(1) $M$ is a compact, connected Kähler manifold;

(2) $M$ is the complement of a (central) complex hyperplane arrangement;

(3) $M$ is a closed, connected, differentiable manifold supporting a free action by a compact, connected real Lie group $K$, and the orbit space $M / K$ is formal in the sense of Sullivan [32].

Here $E(M)=\mathscr{E}(M) \cup\left\{f_{0}\right\}$, where $f_{0}$ realizes abf: $\pi \rightarrow \pi_{\mathrm{abf}}$ and the set $\mathscr{E}(M)$ consists of all admissible maps in the first two cases, and the projection $M \rightarrow M / K$ in the third.

The common strategy of proof is to choose appropriate uniform ACDGA models for the family $E(M)$ and apply Theorem 1.1 to replace topological by infinitesimal equalities. In turn, the latter equalities are verified using results from [22] for parts (1)-(2) and from [28] for part (3).

Compact Kähler manifolds and complements of complex hyperplane arrangements provide highly non-trivial examples of uniform formality (over $\mathbb{k}=\mathbb{R}$ or $\mathbb{C}$ ) with respect to finite families of maps. In Proposition 7.4, we reinterpret the main result from [7] in the following form: $\Omega_{\mathbb{k}}(f) \simeq H^{\bullet}(f, \mathbb{k})$ in ACDGA, uniformly with respect to an arbitrary finite family of holomorphic maps between compact Kähler manifolds. Similarly, we recast in Proposition 9.3 the main result of [12], as follows: $\Omega_{\mathbb{k}}(f) \simeq H^{\bullet}(f, \mathbb{K})$ in ACDGA, uniformly with respect to the family $E\left(M_{\mathscr{A}}\right)$, for any central complex hyperplane arrangement $\mathscr{A}$ in 
$\mathbb{C}^{3}$ with complement $M_{\mathscr{A}}$. In this way, we are able to apply Theorem 1.1 in order to prove parts (1)-(2) of Theorem 1.3.

For part (3), let $N=M / K$ be the orbit space of the free $K$-action on $M$, and let $f: M \rightarrow$ $N$ be the projection map of the resulting principal $K$-bundle. Assuming that $N$ has a finite model $A_{N}$ over a field $\mathbb{k}$ of characteristic 0 , we construct in Proposition 8.2 a finite model $A_{M}$ for $M$ and an ACDGA map $\Phi_{f}: A_{N} \rightarrow A_{M}$ such that $\Omega_{\mathbb{k}}(f) \simeq \Phi_{f}$ in ACDGA. In the case when $N$ is formal, we may take $A_{N}=\left(H^{\bullet}(N, \mathbb{k}), d=0\right)$. Applying now Theorems 1.1 and 1.2 completes the proof of Theorem 1.3(3).

Further applications of the techniques that go into proving the above results can be found in our recent preprint [29]. In particular, in the context of Theorem 1.3, parts (1)(2), but for an arbitrary complex linear algebraic group $G$, it is shown in [29, Theorem 1.1(2)] that the germs $f_{\sharp}^{!} \operatorname{Hom}\left(\pi_{f}, G\right)_{(1)}$ and $g_{\sharp}^{!} \operatorname{Hom}\left(\pi_{g}, G\right)_{(1)}$ from decomposition (1.3) intersect only at the origin, provided the maps $f, g \in \mathscr{E}(M)$ are distinct. This transversality property is a substantial non-abelian extension of the corresponding rank 1 result, proved in [10] in the case when $\iota$ is the standard isomorphism $\mathbb{C}^{\times} \stackrel{\simeq}{\longrightarrow} \mathrm{GL}_{1}(\mathbb{C})$.

1.4. Formal maps and regular maps. The uniformity property for one-element families of maps may be verified in two further classes of examples: formal maps between formal spaces, and regular maps between quasi-projective manifolds.

By definition, a continuous map $f: X \rightarrow Y$ is formal over $\mathbb{k}$ if it is modeled in CDGA by the morphism $H^{\bullet}(f, \mathbb{k}): H^{\bullet}(Y, \mathbb{k}) \rightarrow H^{\bullet}(X, \mathbb{k})$, cf. [32, 34]. In Proposition 3.4, we prove the following: If $f$ is formal over $\mathbb{k}$ and $H^{1}(f, \mathbb{k})$ is injective, then $\Omega_{\mathbb{k}}(f) \simeq H^{\bullet}(f, \mathbb{k})$ in $\mathbb{k}$-ACDGA. Applying Theorem 1.1 yields relevant information (summarized in Proposition 6.8) on the map induced by $f$ between the corresponding embedded jump loci.

To state the quasi-projective analogue of the formality property, we need to recall from $[23,5]$ some relevant facts. Every quasi-projective manifold $M$ is of the form $\bar{M} \backslash D$, where $\bar{M}$ is a smooth, projective variety and $D$ is a normal-crossing divisor in $\bar{M}$. A regular map between two such manifolds, $f: M \rightarrow M^{\prime}$, is induced by a regular map $\bar{f}: \bar{M} \rightarrow \bar{M}^{\prime}$ with the property that $\bar{f}^{-1}\left(D^{\prime}\right) \subseteq D$. The manifold $M$ admits as a finite CDGA model over $\mathbb{C}$ Morgan's Gysin model $\operatorname{MG}(\bar{M}, D)$. Furthermore, the regular map $f$ is modeled in $\mathbb{C}$-CDGA by a certain map $\Phi(\bar{f}): \operatorname{MG}\left(\bar{M}^{\prime}, D^{\prime}\right) \rightarrow \operatorname{MG}(\bar{M}, D)$.

In Proposition 9.1, we use relative Sullivan models for CDGA maps to improve on these known facts, as follows. Let $f: M \rightarrow M^{\prime}$ be a regular map between quasi-projective manifolds, and let $\bar{f}:(\bar{M}, D) \rightarrow\left(\bar{M}^{\prime}, D^{\prime}\right)$ be an extension as above. If $H^{1}(f)$ is injective, then $\Omega_{\mathbb{C}}(f) \simeq \Phi(\bar{f})$ in $\mathbb{C}$-ACDGA. We indicate in Remark 9.2 some possible applications of this result.

1.5. Conventions. All spaces are assumed to be path-connected. The default coefficient ring is a field $\mathbb{k}$ of characteristic 0 . (When speaking about analytic germs and analytic algebras, $\mathbb{k}$ will be either $\mathbb{R}$ or $\mathbb{C}$.) Graded $\mathbb{k}$-vector spaces are non-negatively graded. 


\section{Artin APPRoXimation}

Our approach to naturality properties of cohomology jump loci is based on (simultaneous) Artin approximation, using the book Tougeron [33] as a basic reference. We start by proving a general result of this type.

Given a local ring $(R, \mathrm{~m})$, we denote by gr. $(R)$ the associated graded ring with respect to the m-adic filtration. The m-adic completion of $R$ will be denoted by $\widehat{R}$. If $I \subset R$ is an ideal, $\hat{I}$ will stand for the extended ideal $\widehat{R} \cdot I$ of $\widehat{R}$. Morphisms between local rings are assumed to be local.

We will use M. Artin's theorem on approximating formal power series solutions of analytic equations by convergent power series (see [33, III.4]) in the following form.

Theorem 2.1. Let $R$ and $\bar{R}$ be two analytic algebras, and let $\left\{I_{k}\right\}_{k \in F}$ and $\left\{\bar{I}_{k}\right\}_{k \in F}$ be two finite families of proper ideals in these algebras. Suppose $\alpha: \widehat{R} \rightarrow \widehat{\bar{R}}$ is a morphism sending $\widehat{I}_{k}$ to $\widehat{\bar{I}}_{k}$ for all $k$. There is then a morphism $a: R \rightarrow \bar{R}$ such that $a\left(I_{k}\right) \subset \bar{I}_{k}$ for all $k$ and $\operatorname{gr}_{1}(a)=\operatorname{gr}_{1}(\alpha)$.

The next lemma will also be useful in the sequel.

Lemma 2.2. Let $R$ and $\bar{R}$ be two analytic algebras, and let $I \subset R$ and $\bar{I} \subset \bar{R}$ be two proper ideals. Suppose $a: R \rightarrow \bar{R}$ is a morphism that sends I to $\bar{I}$, and $\alpha: \widehat{R} \rightarrow \widehat{\bar{R}}$ is an isomorphism such that $\alpha(\widehat{I})=\widehat{\bar{I}}$ and $\operatorname{gr}_{1}(a)=\operatorname{gr}_{1}(\alpha)$. Then $a$ is an isomorphism and $a(I)=\bar{I}$.

Proof. It follows from [33, III.5] that a morphism of analytic algebras with isomorphic completions must be an isomorphism, provided the given morphism induces a surjection on $\mathrm{gr}_{1}$. Consequently, both $a: R \rightarrow \bar{R}$ and the induced morphism, $a^{\prime}: R / I \rightarrow \bar{R} / \bar{I}$, are isomorphisms, and the claim follows.

We are now ready to describe the setup for our approximation result. Let $\left\{\phi_{i}: R \rightarrow\right.$ $\left.R_{i}\right\}_{i \in E}$ and $\left\{\bar{\phi}_{i}: \bar{R} \rightarrow \bar{R}_{i}\right\}_{i \in E}$ be two families of epimorphisms between analytic algebras, indexed by the same finite set $E$. Furthermore, let $\left\{I^{j} \subseteq R\right\}_{j \in F},\left\{\bar{I}^{j} \subseteq \bar{R}\right\}_{j \in F},\left\{I_{i}^{j} \subseteq R_{i}\right\}_{j \in F}$, and $\left\{\bar{I}_{i}^{j} \subseteq \bar{R}_{i}\right\}_{j \in F}$ be families of ideals in the respective analytic algebras, indexed by the same finite set $F$. Finally, let $\alpha: \widehat{R} \cong \widehat{\bar{R}}$ and $\left\{\alpha_{i}: \widehat{R}_{i} \cong \widehat{\bar{R}}_{i}\right\}_{i \in E}$ be isomorphisms between the respective completions.

Proposition 2.3. In the above setup, assume the following conditions hold, for all $i \in E$ and $j \in F$ (as the case may be):

(1) $I^{j} \neq R \Leftrightarrow \bar{I}^{j} \neq \bar{R}, I_{i}^{j} \neq R_{i} \Leftrightarrow \bar{I}_{i}^{j} \neq \bar{R}_{i}, I^{j}=R \Rightarrow I_{i}^{j}=R_{i}, \bar{I}^{j}=\bar{R} \Rightarrow \bar{I}_{i}^{j}=\bar{R}_{i}$;

(2) $\alpha\left(\widehat{I}^{j}\right)=\widehat{\bar{I}}^{j}$;

(3) $\alpha_{i}\left(\widehat{I}_{i}^{j}\right)=\widehat{\bar{I}}_{i}^{j}$; 
(4) $\hat{\bar{\phi}}_{i} \circ \alpha=\alpha_{i} \circ \hat{\phi}_{i}$.

There exist then isomorphisms $a: R \stackrel{\longrightarrow}{\longrightarrow}$ and $\left\{a_{i}: R_{i} \stackrel{\simeq}{\longrightarrow} \bar{R}_{i}\right\}_{i \in E}$ such that

(i) $a\left(I^{j}\right)=\bar{I}^{j}$ for all $j \in F$;

(ii) $a_{i}\left(I_{i}^{j}\right)=\bar{I}_{i}^{j}$ for all $i \in E$ and $j \in F$;

(iii) $\bar{\phi}_{i} \circ a=a_{i} \circ \phi_{i}$ for all $i \in E$.

Proof. Without loss of generality, we may assume all ideals in sight are proper, replacing if need be the set $F$ by subsets $F_{0} \subseteq F$ and $F_{i} \subseteq F_{0}$ for $i \in E$. For each $i \in E$, put $K_{i}=\operatorname{ker}\left(\phi_{i}\right)$ and $\bar{K}_{i}=\operatorname{ker}\left(\bar{\phi}_{i}\right)$, and pick proper ideals, $\left\{J_{i}^{j} \subset R\right\}_{j \in F_{i}}$ and $\left\{\bar{J}_{i}^{j} \subset \bar{R}\right\}_{j \in F_{i}}$, such that $\phi_{i}\left(J_{i}^{j}\right)=I_{i}^{j}$ and $\bar{\phi}_{i}\left(\bar{J}_{i}^{j}\right)=\bar{I}_{i}^{j}$. We claim that it is enough to find a morphism $a: R \rightarrow \bar{R}$ such that
(a) $\operatorname{gr}_{1}(a)=\operatorname{gr}_{1}(\alpha)$
(b) $a\left(K_{i}\right) \subseteq \bar{K}_{i}$, for all $i \in E$;
(c) $a\left(I^{j}\right) \subseteq \bar{I}^{j}$, for all $j \in F_{0}$;
(d) $a\left(J_{i}^{j}\right) \subseteq \bar{J}_{i}^{j}+\bar{K}_{i}$, for all $(i, j) \in E \times F_{i}$.

Indeed, by (b), the morphism $a: R \rightarrow \bar{R}$ induces morphisms $a_{i}: R_{i} \rightarrow \bar{R}_{i}$ for all $i \in E$. In view of (a), we may apply Lemma 2.2 and deduce that the map $a$ is an isomorphism. By construction, property (iii) is satisfied. By assumption, equality (4) holds, and so $\alpha\left(\widehat{K}_{i}\right)=\widehat{\bar{K}}_{i}$. Again by Lemma 2.2, the maps $a_{i}$ must be isomorphisms, for all $i \in E$. In view of (c), we may also apply Lemma 2.2 to each of the ideals $I^{j} \subset R$ and $\bar{I}^{j} \subset \bar{R}$ for $j \in F_{0}$, and deduce that $a\left(I^{j}\right)=\bar{I}^{j}$, thereby verifying property (i).

Finally, to verify property (ii), we apply Lemma 2.2 to the ideals $I_{i}^{j}=\left(J_{i}^{j}+K_{i}\right) / K_{i} \subset$ $R / K_{i}=R_{i}$ and $\bar{I}_{i}^{j}=\left(\bar{J}_{i}^{j}+\bar{K}_{i}\right) / \bar{K}_{i} \subset \bar{R} / \bar{K}_{i}=\bar{R}_{i}$ for $(i, j) \in E \times F_{i}$. We know from (b) and (d) that the morphisms $a_{i}: R_{i} \rightarrow \bar{R}_{i}$ preserve these ideals. The fact that the isomorphisms $\alpha_{i}$ identify the completions of these ideals follows from their construction, together with assumption (3). Moreover, since $a_{i}$ is induced by $a$ and $\alpha_{i}$ is induced by $\alpha$, property (a) implies that $\operatorname{gr}_{1}\left(a_{i}\right)=\operatorname{gr}_{1}\left(\alpha_{i}\right)$. Thus, Lemma 2.2 applies once again to show that $a_{i}\left(I_{i}^{j}\right)=\bar{I}_{i}^{j}$. This completes the verification of our claim.

Assumptions (2)-(4) insure that the map $\alpha: \widehat{R} \cong \widehat{\bar{R}}$ is a formal series solution of the analytic system (b)-(d). Applying now Theorem 2.1 completes the proof.

\section{Algebraic MOdels of SPACES AND MAPS}

The rational homotopy theory of Quillen [30], as reinterpreted by Sullivan in [32], provides a very useful mechanism for studying topological properties of spaces and continuous maps by considering commutative differential graded algebra (for short, CDGA) models for them. In this section, we review the basics of this theory, and draw some consequences in the formal case. 
3.1. $q$-connectivity and $q$-equivalences. We start with some basic terminology, related to connectivity properties of spaces and CDGAs. Fix $0 \leqslant q \leqslant \infty$, usually to be omitted from notation when $q=\infty$. Let $\psi: C^{\bullet} \rightarrow C^{\bullet}$ be a morphism of graded vector spaces. We say $\psi$ is $q$-connected if it is an isomorphism in degrees up to $q$ and a monomorphism in degree $q+1$.

When such a map $\psi$ is a $q$-connected morphism of cochain complexes, it is straightforward to check that the induced morphism in cohomology, $H^{\bullet}(\psi): H^{\bullet}(C) \rightarrow H^{\bullet}\left(C^{\prime}\right)$, is again $q$-connected. Cochain maps inducing a $q$-connected map in cohomology will be called $q$-equivalences. For $q=\infty$, these maps are also called quasi-isomorphisms in the literature.

We say that a commutative graded algebra (for short, a CGA) $A^{\bullet}$ is connected if $A^{0}$ is the $\mathbb{k}$-span of the unit 1 (and thus $A^{0}=\mathbb{k}$ ). If $A$ is a connected CDGA, then clearly its cohomology algebra, $H^{\bullet}(A)$, is again connected.

This terminology is inspired by algebraic topology, where a continuous map $f: X \rightarrow Y$ between two topological spaces is said to be $q$-connected if it induces isomorphisms on homotopy groups up to degree $q$ and an epimorphism in degree $q+1$. By Hurewicz's theorem, the induced map in cohomology, $H^{\bullet}(f): H^{\bullet}(Y) \rightarrow H^{\bullet}(X)$, is also $q$-connected. Note that the map $X \rightarrow\{\mathrm{pt}\}$ is $q$-connected if and only if the space $X$ is $q$-connected.

Finally, let $C$ be a subcategory of the category of cochain complexes, for instance, CDGA or the category of differential graded Lie algebras, DGL. Two objects in this category, $C$ and $C^{\prime}$, have the same q-type (denoted $C \simeq_{q} C^{\prime}$ ) if they can be connected in C by a zig-zag of $q$-equivalences.

3.2. CDGA models for spaces. We now review the construction and some basic properties of CDGA models of spaces, following [32, 23, 17, 19, 13, 9]. We will denote by $\Omega_{\mathbb{k}}^{\bullet}(X)$ Sullivan's de Rham algebra of a topological space $X$, constructed by using differential forms with $\mathbb{k}$-polynomial coefficients on standard simplices, see [32]. The resulting functor Top $\rightarrow \mathbb{k}$-CDGA has, among other things, the property that $H^{\bullet}\left(\Omega_{\mathbb{k}}^{\bullet}(X)\right) \cong H^{\bullet}(X, \mathbb{k})$, as graded $\mathbb{k}$-algebras.

To define monodromy representations of flat connections (over $\mathbb{k}=\mathbb{R}$ or $\mathbb{C}$ ), we will also need the similar CDGA $\Omega^{\bullet}(X, \mathbb{k})$, constructed from usual smooth $\mathbb{k}$-forms. It is known that $\Omega^{\bullet}(X, \mathbb{k})$ has the same $\infty$-type as the sub-CDGA $\Omega_{\mathbb{k}}^{\bullet}(X)$, in a natural way.

Let $A$ be a CDGA. For $0 \leqslant q \leqslant \infty$, we say that $A$ is a $q$-model for the space $X$ if $\Omega_{\mathbb{k}}(X) \simeq_{q} A$. We also say that $X$ is $q$-finite if it has the homotopy type of a connected $\mathrm{CW}$-complex with finite $q$-skeleton. Similarly, we say that $A$ is $q$-finite if it is connected and $\operatorname{dim} \bigoplus_{i \leqslant q} A^{i}<\infty$. Once again, we shall omit $q$ from the notation when $q=\infty$.

The category ACDGA of augmented, commutative differential graded algebras has objects $(A, \varepsilon)$, where the augmentation map $\varepsilon: A \rightarrow \mathbb{K}$ is a morphism of CDGAs, while the morphisms in this category are the CDGA maps commuting with augmentations. When $X$ is a pointed space, both $\Omega^{\bullet}(X, \mathbb{K})$ and $\Omega_{\mathbb{k}}^{\bullet}(X)$ become ACDGAs, again in a natural way. 
A connected CDGA $A$ has a unique augmentation map, sending $A^{+}$to 0 , and the unit to 1. Moreover, for every augmented CDGA $A^{\prime}$, we have that

$$
\operatorname{Hom}_{\mathrm{ACDGA}}\left(A, A^{\prime}\right)=\operatorname{Hom}_{\mathrm{CDGA}}\left(A, A^{\prime}\right) \text {. }
$$

3.3. Hirsch extensions and relative minimal models. Let $U^{\bullet}=\bigoplus_{i \geqslant 1} U^{i}$ be a positively graded $\mathbb{k}$-vector space. The free commutative graded algebra on $U$, denoted by $\wedge U^{\bullet}$, is the tensor product of the symmetric graded algebra on $U^{\text {even }}$ and the exterior graded algebra on $U^{\text {odd }}$. We say that a CDGA is free if the underlying CGA has this property. Since $\wedge U^{\bullet}$ is connected, it has a unique augmentation, denoted by $\varepsilon_{U}$.

Let $A=\left(A^{\bullet}, d_{A}\right)$ be a CDGA, and denote by $Z^{\bullet}(A)$ the graded vector space of cocycles. Given a finite-dimensional graded vector space $U^{\bullet}$, and a degree 1 linear map, $\tau: U^{\bullet} \rightarrow$ $Z^{\bullet+1}(A)$, we denote by $\left(A \otimes_{\tau} \wedge U, d\right)$ the corresponding Hirsch extension. By definition, this is the CDGA whose underlying CGA is $A^{\bullet} \otimes \wedge U^{\bullet}$, and whose differential restricts to $d_{A}$ on $A$ and to $\tau$ on $U$. If $A$ is an ACDGA with augmentation $\varepsilon_{A}$, then $A \otimes_{\tau} \wedge U$ is also an ACDGA, with augmentation $\varepsilon_{A} \otimes \varepsilon_{U}$. The Hirsch extension depends only on the map $[\tau]: U^{\bullet} \rightarrow H^{\bullet+1}(A)$, in the following sense: if $[\tau]=\left[\tau^{\prime}\right]$, then $A \otimes_{\tau} \wedge U \cong A \otimes_{\tau^{\prime}} \wedge U$ in CDGA, via an isomorphism extending $\operatorname{id}_{A}$. When $\operatorname{dim} U=1$, we speak of an 'elementary' Hirsch extension.

A relative Sullivan algebra with base $B$ is a direct limit of elementary Hirsch extensions, starting from the CDGA $B$. When the base is $\mathbb{k}$, concentrated in degree 0 , we simply speak of a Sullivan algebra. Such a CDGA is necessarily of the form $\mathscr{M}=\left(\bigwedge U^{\bullet}, d\right)$. If $\operatorname{im}(d) \subseteq \bigwedge^{\geqslant 2} U$, we say $\mathscr{M}$ is a minimal Sullivan algebra. If, moreover, all Hirsch extensions have degree at most $q$, the CDGA $\mathscr{M}$ is said to be $q$-minimal.

A $q$-minimal model map for a CDGA $A$ is a $q$-equivalence $\rho: \mathscr{M}_{q} \rightarrow A$, with $\mathscr{M}_{q}$ a $q$ minimal Sullivan algebra. Any CDGA $A$ whose cohomology algebra is connected admits a $q$-minimal model map. If $\rho^{\prime}: \mathscr{M}_{q}^{\prime} \rightarrow A$ is another $q$-minimal model map for $A$, then $\mathscr{M}_{q}^{\prime}$ and $\mathscr{M}_{q}$ are isomorphic in CDGA. Consequently, if $A$ and $\bar{A}$ are two CDGAs with connected homology, then $A \simeq_{q} \bar{A}$ in CDGA if and only if there is a $q$-minimal CDGA $\mathscr{M}_{q}$, and a short zig-zag of $q$-equivalences in CDGA of the form

$$
A \stackrel{\rho}{\longleftarrow} \mathscr{M}_{q} \stackrel{\bar{\rho}}{\longrightarrow} \bar{A} .
$$

Recall that a relative Sullivan algebra with base $B$ is a CDGA of the form $A=(B \otimes$ $\bigwedge U, d)$. When $B$ is an augmented algebra, with augmentation ideal $\widetilde{B}:=\operatorname{ker}\left(\varepsilon_{B}\right)$, the quotient CDGA, $A /(\widetilde{B} \otimes \bigwedge U)=(\bigwedge U, \bar{d})$, is called the fiber of $A$. Following $[17,13]$, we say that $A$ is a minimal Sullivan algebra in the relative sense if the fiber is minimal. Allowing also degree 0 Hirsch extensions, we may speak of weak relative Sullivan (minimal) algebras.

Let $\Phi: B \rightarrow C$ be a CDGA map, and assume $B$ is augmented and $H^{\bullet}(B)$ is connected. A relative minimal model map for $\Phi$ is an $\infty$-equivalence of CDGAs, $h: \mathscr{M} \rightarrow C$, where 
$\mathscr{M}=(B \otimes \bigwedge U, d)$ is a relative minimal Sullivan algebra and $\left.h\right|_{B}=\Phi$. If $\Phi$ is a 0 equivalence, then $\Phi$ admits a relative minimal model map; moreover, any two such maps, $h$ and $h^{\prime}$, have isomorphic fibers, see [13, §14]. In fact, existence and uniqueness also hold in the weak sense, assuming only that $H^{0}(\Phi)$ is an isomorphism, see [17, Ch. 6]. In particular, if $\Phi: B \rightarrow C$ is a 0 -equivalence and $h: \mathscr{M} \rightarrow C$ is any relative minimal model map for $\Phi$ in the weak sense, then necessarily the fiber of $\mathscr{M}$ is connected. Hence, if $\Phi$ is an ACDGA map, then $\mathscr{M}$ is canonically augmented and both $h$ and the inclusion $B \hookrightarrow \mathscr{M}$ preserve augmentations.

3.4. Homotopies and equivalences. Let $\bigwedge(t, d t)$ be the free, contractible CDGA generated by $t$ in degree 0 and $d t$ in degree 1 . For each $s \in \mathbb{k}$, let ev $: \wedge(t, d t) \rightarrow \mathbb{k}$ be the CDGA map sending $t$ to $s$ and $d t$ to 0 . This map induces another CDGA map,

$$
\mathrm{Ev}_{s}=\mathrm{id} \otimes \mathrm{ev}_{s}: A \otimes \bigwedge(t, d t) \rightarrow A \otimes \mathbb{k}=A .
$$

Definition 3.1. Two CDGA maps $\psi_{0}, \psi_{1}: A \rightarrow A^{\prime}$ are said to be homotopic (in CDGA) if there is a CDGA map $\Psi: A \rightarrow A^{\prime} \otimes \wedge(t, d t)$ such that $\mathrm{Ev}_{s} \circ \Psi=\psi_{s}$ for $s=0,1$. Likewise, two ACDGA maps $\psi_{0}$ and $\psi_{1}$ as above are homotopic (in ACDGA) if the homotopy $\Psi$ also satisfies $\mathrm{Ev}^{\prime} \circ \Psi=\varepsilon$, where $\mathrm{Ev}^{\prime}$ denotes the CDGA map $\varepsilon^{\prime} \otimes \mathrm{id}: A^{\prime} \otimes \wedge(t, d t) \rightarrow \wedge(t, d t)$.

Plainly, equality of maps implies homotopy, in both categories. Note that augmented homotopy is strictly stronger than homotopy. Another useful remark is that homotopic maps in CDGA induce the same map in cohomology.

Denote by $\mathrm{ACDGA}_{0}$ the full subcategory of ACDGA whose objects have connected cohomology. Fix an integer $q \geqslant 0$.

Definition 3.2. An elementary q-equivalence in $\mathrm{ACDGA}_{0}$ between two $\mathrm{ACDGA}_{0}$-morphisms $\Phi_{0}: A_{0}^{\prime} \rightarrow A_{0}$ and $\Phi_{1}: A_{1}^{\prime} \rightarrow A_{1}$ consists of two ACDGA maps, $\psi: A_{1} \rightarrow A_{0}$ and $\psi^{\prime}: A_{1}^{\prime} \rightarrow$ $A_{0}^{\prime}$, both of which are $q$-equivalences, and such that $\psi \circ \Phi_{1}$ is homotopic to $\Phi_{0} \circ \psi^{\prime}$ in ACDGA. We denote by $\simeq_{q}$ the associated equivalence relation between morphisms in $\mathrm{ACDGA}_{0}$.

In other words, if $\Phi: A^{\prime} \rightarrow A$ and $\bar{\Phi}: B^{\prime} \rightarrow B$ are two such morphisms, we say that $\Phi \simeq_{q} \bar{\Phi}$ in $\mathrm{ACDGA}_{0}$ if there are two zig-zags, $Z$ and $Z^{\prime}$, of $q$-equivalences in ACDGA, and ACDGA maps $\Phi_{1}, \ldots, \Phi_{\ell-1}$ such that the following diagram commutes, up to augmented homotopy:

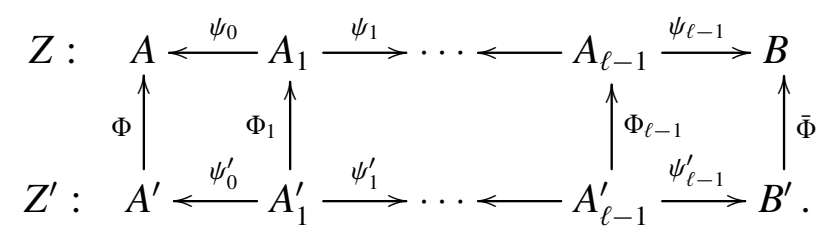

Forgetting augmentations in Definition 3.2, we obtain the equivalence relation $\simeq_{q}$ between maps in $\mathrm{CDGA}_{0}$. When $q=\infty$, we will simply write this as $\Phi \simeq \bar{\Phi}$. 
Lemma 3.3. Let $A$ and $\bar{A}$ be two ACDGAs. Assume $H^{\bullet}(A)$ is connected, and let $\rho: \mathscr{M}_{q} \rightarrow$ A be a q-minimal CDGA model map as above. Then $A \simeq_{q} \bar{A}$ in CDGA if and only if there is a short zig-zag of q-equivalences in ACDGA as in (3.2).

Proof. By the discussion from $\$ 3.3$, we have that $A \simeq_{q} \bar{A}$ in CDGA if and only if $A$ and $\bar{A}$ share the same $q$-minimal model $\mathscr{M}_{q}$. The fact that $\mathscr{M}_{q}$ is connected takes care of the augmentations.

3.5. Formal spaces and maps. We conclude this section with some formality notions, for both spaces and maps, as well as models thereof. To start with, we say that a CDGA $A$ is $q$-formal if $\left(A^{\bullet}, d\right) \simeq_{q}\left(H^{\bullet}(A), d=0\right)$ in CDGA. Clearly, $q$-formality implies $p$-formality, for all $p \leqslant q$. By definition, a space $X$ is $q$-formal over $\mathbb{k}$ if $\Omega_{\mathbb{k}}(X)$ has this property. A $q$-finite, $q$-formal space $X$ has the $q$-finite $q$-model $\left(H^{\bullet}(X), d=0\right)$. As before, we will mostly omit $q$ from notation when $q=\infty$. Compact Kähler manifolds are well-known to be formal, by the main result from [7].

Following [32, 7, 34], we say that a morphism $\Phi: A^{\prime} \rightarrow A$ in $\mathrm{CDGA}_{0}$ is formal if there is a diagram consisting of two elementary equivalences in $\mathrm{CDGA}_{0}$,

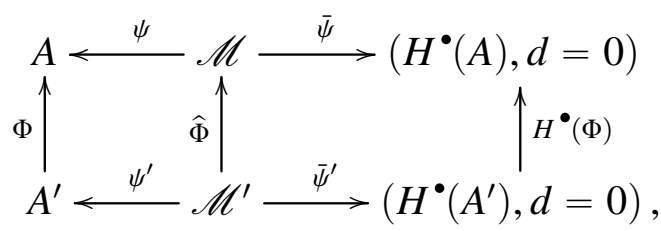

such that both $\mathscr{M}$ and $\mathscr{M}^{\prime}$ are minimal Sullivan algebras. Furthermore, we say that a continuous map $f: X \rightarrow X^{\prime}$ is formal (over $\mathbb{k}$ ) if the induced morphism between Sullivan de Rham models, $\Omega_{\mathbb{k}}(f): \Omega_{\mathbb{k}}\left(X^{\prime}\right) \rightarrow \Omega_{\mathbb{k}}(X)$, has this property.

Proposition 3.4. Let $f: X \rightarrow X^{\prime}$ be a continuous map between pointed spaces. Assume that $f$ is formal over $\mathbb{k}$, and $H^{1}(f)$ is injective. Then $\Omega(f) \simeq H^{\bullet}(f)$ in $\mathbb{k}-\mathrm{ACDGA}_{0}$.

Proof. In [34, II.3], Vigué-Poirrier uses the formality of the map $f$ to construct a commuting diagram in CDGA of the form

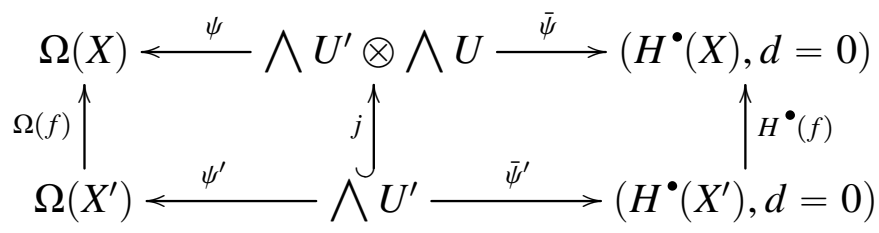

where all horizontal arrows are $\infty$-equivalences and $j$ is the canonical inclusion. Moreover, $\psi^{\prime}$ and $\bar{\psi}^{\prime}$ are minimal model maps (in particular, $\bigwedge U^{\prime}$ is connected), and $\bigwedge U^{\prime} \otimes$ $\bigwedge U$ is a relative minimal Sullivan algebra in the weak sense, with base $\bigwedge U^{\prime}$.

The injectivity assumption on $H^{1}(f)$ implies that $H^{\bullet}(f) \circ \bar{\psi}^{\prime}$ is a 0 -equivalence. From the discussion in $\S 3.3$, we deduce that $U^{0}=0$. This shows that $\bigwedge U^{\prime} \otimes \wedge U$ is also connected. Hence, (3.6) is a commuting diagram in $\mathrm{ACDGA}_{0}$, and our claim follows. 


\section{DeFORMATION THEORY OF REPRESENTATION VARIETIES}

Following Goldman-Millson [15] and Manetti [21], we recall, in a convenient form, two basic properties of the deformation functor associated to a differential graded Lie algebra (DGL for short). We then apply these techniques to the representation varieties of discrete groups.

4.1. Deformation functors. We denote by Art the category of Artinian local $\mathbb{k}$-algebras. Given such an algebra $\left(A, m_{A}\right)$ and a differential graded Lie algebra $L$, we consider the (nilpotent) DGL $L \otimes \mathrm{m}_{\mathrm{A}}$, and the set of solutions (called flat connections) to the MaurerCartan equation,

$$
\mathscr{F}\left(L \otimes \mathrm{m}_{\mathrm{A}}\right)=\left\{\omega \in L^{1} \otimes \mathrm{m}_{\mathrm{A}} \mid d \omega+\frac{1}{2}[\omega, \omega]=0\right\},
$$

with basepoint $0 \in \mathscr{F}\left(L \otimes \mathrm{m}_{\mathrm{A}}\right)$. Clearly, this construction is bifunctorial. Let

$$
\mathscr{G}\left(L \otimes \mathrm{m}_{\mathrm{A}}\right)=\exp \left(L^{0} \otimes \mathrm{m}_{\mathrm{A}}\right)
$$

be the (Campbell-Hausdorff) gauge group of the nilpotent Lie algebra $L^{0} \otimes \mathrm{m}_{\mathrm{A}}$, with underlying set $L^{0} \otimes \mathrm{m}_{\mathrm{A}}$. This group acts bifunctorially on $\mathscr{F}\left(L \otimes \mathrm{m}_{\mathrm{A}}\right)$ by

$$
\exp (\alpha) \cdot \omega=\omega+\sum_{n=0}^{\infty} \frac{\operatorname{ad}(\alpha)^{n}}{(n+1) !}([\alpha, \omega]-d \alpha)
$$

The deformation functor, $\operatorname{Def}_{L}:$ Art $\rightarrow$ Set, is defined by

$$
\operatorname{Def}_{L}(\mathrm{~A})=\mathscr{F}\left(L \otimes \mathrm{m}_{\mathrm{A}}\right) / \mathscr{G}\left(L \otimes \mathrm{m}_{\mathrm{A}}\right) .
$$

It is readily seen that every DGL-morphism $\psi: L \rightarrow L^{\prime}$ induces a natural transformation, $\operatorname{Def}_{\psi}: \operatorname{Def}_{L} \rightarrow \operatorname{Def}_{L^{\prime}}$.

We now may state the Deligne-Schlesinger-Stasheff theorem, as recorded and proved in [15, Thm. 2.4].

Theorem 4.1. If $L \simeq_{1} L^{\prime}$ in DGL, then the deformation functors $\operatorname{Def}_{L}$ and $\operatorname{Def}_{L^{\prime}}$ are naturally isomorphic.

4.2. Homotopy invariance. The homotopy relation in DGL takes the following form. Given a DGL $L$, let us form the DGL $L \otimes \wedge(t, d t)$, endowed with the canonical tensor product structure. For each $s \in \mathbb{k}$, we defined in $\$ 3.4$ an evaluation CDGA map, $\mathrm{ev}_{s}: \wedge(t, d t) \rightarrow \mathbb{k}$, which sends $t \mapsto s$ and $d t \mapsto 0$. Proceeding as before, we extend this map to a DGL map, $\mathrm{Ev}_{s}=\mathrm{id} \otimes \mathrm{ev}_{s}: L \otimes \wedge(t, d t) \rightarrow L \otimes \mathbb{K}=L$.

Two DGL maps $\psi_{0}, \psi_{1}: L \rightarrow L^{\prime}$ are said to be homotopic if there is a DGL map $\Psi: L \rightarrow$ $L^{\prime} \otimes \bigwedge(t, d t)$ such that $\mathrm{Ev}_{s} \circ \Psi=\psi_{s}$ for $s=0,1$. The notion of homotopy between DGL maps is related to deformation functors via the following basic result of Manetti [21, Thm. 5.5]. 
Theorem 4.2. Let $L$ be a DGL, and let $A$ be a local Artin algebra. Two flat connections $\beta_{0}, \beta_{1} \in \mathscr{F}\left(L \otimes \mathrm{m}_{A}\right)$ are equal in $\operatorname{Def}_{L}(A)$ if and only if there is a flat connection $\omega \in$ $\mathscr{F}\left(L \otimes \bigwedge(t, d t) \otimes \mathrm{m}_{A}\right)$ such that $\left(\mathrm{Ev}_{s} \otimes \mathrm{id}\right) \omega=\beta_{s}$ for $s=0,1$.

This theorem has an immediate corollary, which will be useful in the sequel.

Corollary 4.3. If $\psi_{0}, \psi_{1}: L \rightarrow L^{\prime}$ are homotopic in DGL, then $\operatorname{Def}_{\psi_{0}}=\operatorname{Def}_{\psi_{1}}$.

4.3. Deformation theory of CDGAs. We consider now the bifunctor CDGA $\times$ Lie $\rightarrow$ DGL which associates to a CDGA $A$ and a Lie algebra $\mathfrak{g}$ the DGL

$$
L=A \otimes \mathfrak{g},
$$

endowed with the canonical tensor product structure $\left[a \otimes g, a^{\prime} \otimes g^{\prime}\right]=a a^{\prime} \otimes\left[g, g^{\prime}\right]$ and differential $\partial(a \otimes g)=d a \otimes g$.

We will also need an augmented version of this construction. Given an augmented CDGA $(A, \varepsilon)$ and a Lie algebra $\mathfrak{g}$, we denote by $\widetilde{A}=\operatorname{ker}(\varepsilon)$ the augmentation differential ideal, and we consider the sub-DGL $\widetilde{L}=\widetilde{A} \otimes \mathfrak{g}$ of the DGL $L=A \otimes \mathfrak{g}$. This construction is again bifunctorial.

Remark 4.4. Given a $q$-equivalence $\psi \in \operatorname{Hom}_{\mathrm{ACDGA}}\left(A, A^{\prime}\right)$, it is easy to check that the induced maps, $\tilde{\psi}: \widetilde{A} \rightarrow \widetilde{A}^{\prime}$ and $\tilde{\psi} \otimes \mathrm{id}: \widetilde{L} \rightarrow \widetilde{L}^{\prime}$, are again $q$-equivalences, provided that both $H^{\bullet}(A)$ and $H^{\bullet}\left(A^{\prime}\right)$ are connected. Consequently, if $A \simeq_{q} A^{\prime}$ in $\operatorname{ACDGA}_{0}$ then $\widetilde{L} \simeq_{q} \widetilde{L}^{\prime}$ in DGL.

Let $\mathfrak{g}$ be a Lie algebra. The proof of the next lemma is straightforward.

Lemma 4.5. $A$ CDGA homotopy, $\Psi: A \rightarrow A^{\prime} \otimes \wedge(t, d t)$, between two maps, $\psi_{0}$ and $\psi_{1}$, induces a DGL homotopy, $\Psi \otimes \mathrm{id}: A \otimes \mathfrak{g} \rightarrow A^{\prime} \otimes \mathfrak{g} \otimes \wedge(t, d t)$, between the maps $\psi_{0} \otimes \mathrm{id}$ and $\psi_{1} \otimes \mathrm{id}$. Moreover, if $\Psi$ is an augmented homotopy, then $\Psi$ induces a DGL homotopy, $\widetilde{\Psi} \otimes \mathrm{id}: \widetilde{A} \otimes \mathfrak{g} \rightarrow \widetilde{A}^{\prime} \otimes \mathfrak{g} \otimes \wedge(t, d t)$, between the maps $\tilde{\psi}_{0} \otimes \mathrm{id}$ and $\tilde{\psi}_{1} \otimes \mathrm{id}$.

4.4. Deformation theory of augmented CDGAs. Our next goal is to relate $q$-types of CDGAs to the deformation theory of ACDGAs. Fix $q \geqslant 1$, and let $Z$ be a zig-zag of $q$ equivalences in ACDGA,

$$
A_{0} \stackrel{\psi_{0}}{\longleftarrow} A_{1} \stackrel{\psi_{1}}{\longrightarrow} \cdots \longleftarrow A_{\ell-1} \stackrel{\psi_{\ell-1}}{\longrightarrow} A_{\ell},
$$

where $H^{0}\left(A_{0}\right)=\mathbb{k} \cdot 1$. By Remark 4.4 and Theorem 4.1, the zig-zag $Z$ induces a natural bijection

$$
\beta_{Z}: \mathscr{F}\left(\widetilde{A}_{\ell} \otimes \mathfrak{g} \otimes \mathrm{m}_{\mathrm{A}}\right) / \mathscr{G}\left(\widetilde{A}_{\ell} \otimes \mathfrak{g} \otimes \mathrm{m}_{\mathrm{A}}\right) \stackrel{\simeq}{\longrightarrow} \mathscr{F}\left(\widetilde{A}_{0} \otimes \mathfrak{g} \otimes \mathrm{m}_{\mathrm{A}}\right) / \mathscr{G}\left(\widetilde{A}_{0} \otimes \mathfrak{g} \otimes \mathrm{m}_{\mathrm{A}}\right)
$$

for all local Artin algebras $A$. It is important to note that, if $Z$ and $Z^{\prime}$ are two different zig-zags of $q$-equivalences connecting $A_{0}$ to $A_{\ell}$, then the bijections $\beta_{Z}$ and $\beta_{Z^{\prime}}$ may also be different. 
Proposition 4.6. Let $\rho: \mathscr{M}_{q} \rightarrow A_{0}$ be a q-minimal model map. There is then a short zig-zag of q-equivalences in ACDGA,

$$
S: A_{0} \stackrel{\rho}{\longleftarrow} \mathscr{M}_{q} \stackrel{\bar{\rho}}{\longrightarrow} A_{\ell},
$$

such that $\beta_{Z}=\beta_{S}$.

Proof. We will construct, by induction on $0 \leqslant i \leqslant \ell$, a collection of $q$-minimal model maps $\rho_{i}: \mathscr{M}_{q} \rightarrow A_{i}$, which form, together with the maps $\psi_{i}$ from (4.6), homotopy-commutative triangles in ACDGA, starting with $\rho_{0}=\rho$. Once this done, we set $\bar{\rho}=\rho_{\ell}$. The equality $\beta_{Z}=\beta_{S}$ then follows from Lemma 4.5 and Corollary 4.3.

For the induction step, we first assume that $\psi_{i}: A_{i} \rightarrow A_{i+1}$. Then we take $\rho_{i+1}=\psi_{i} \circ \rho_{i}$. Finally, suppose that $\psi_{i}: A_{i+1} \rightarrow A_{i}$. The lifting property up to homotopy for CDGA maps also holds for ACDGA maps, and implies that we may find a CDGA map $\rho_{i+1}: \mathscr{M}_{q} \rightarrow A_{i+1}$ such that $\psi_{i} \circ \rho_{i+1}$ is homotopic to $\rho_{i}$ in ACDGA. The fact that $\rho_{i+1}$ must be a $q$-equivalence is easily checked, thereby completing the proof.

4.5. Representation varieties. Let $\pi$ be a discrete group, and let $G$ be a $\mathbb{k}$-linear algebraic group. The set $\operatorname{Hom}(\pi, G)$ of group homomorphisms from $\pi$ to $G$ has a natural structure of an affine scheme. This set depends bi-functorially on $\pi$ and $G$, and has a natural base point, the trivial representation, 1 . Furthermore, $G$ acts by conjugation on $\operatorname{Hom}(\pi, G)$.

Now suppose $\pi$ is a finitely generated group. (Note that the fundamental group $\pi=$ $\pi_{1}(X, x)$ of a pointed CW-space is finitely generated if and only if $X$ is 1-finite.) In this case, the set $\operatorname{Hom}(\pi, G)$ has a natural structure of affine variety, called the $G$-representation variety of $\pi$. Moreover, every homomorphism $\varphi: \pi \rightarrow \pi^{\prime}$ induces an algebraic morphism between the corresponding representation varieties, $\varphi^{!}: \operatorname{Hom}\left(\pi^{\prime}, G\right) \rightarrow \operatorname{Hom}(\pi, G)$. We will come back to this point in Lemma 5.3.

Clearly, the $G$-representation variety of the free group $F_{n}$ is equal to the $n$-fold direct product $G^{n}$. Much is known about the varieties of commuting matrices, for instance, that $\operatorname{Hom}\left(\mathbb{Z}^{2}, \mathrm{GL}_{n}(\mathbb{C})\right)$ is irreducible. Nevertheless, many open questions remain about the precise structure of the varieties $\operatorname{Hom}\left(\mathbb{Z}^{n}, G\right)$, see for instance $[3,1]$ and references therein. Perhaps the most-studied family of representation varieties is that of fundamental groups of closed orientable surfaces $\Sigma_{g}$. For instance, it is known that $\operatorname{Hom}\left(\pi_{1}\left(\Sigma_{g}\right), G\right)$ is connected if $G=\mathrm{SL}_{n}(\mathbb{C})$, and an absolutely irreducible and $\mathbb{Q}$-rational variety if $G=$ $\mathrm{GL}_{n}(\mathbb{C})$, see $[14,31]$.

4.6. Flat connections. The infinitesimal counterpart to the representation varieties is provided by the space of flat connections. Given a CDGA $A$ and a Lie algebra $\mathfrak{g}$, we will denote by $\mathscr{F}(A, \mathfrak{g})$ the set of flat connections on the DGL $A \otimes \mathfrak{g}$. This set behaves bi-functorially, and has a natural basepoint, the trivial flat connection 0 . For a local Artin $\mathbb{K}$-algebra $A$, the gauge group

$$
\mathscr{G}\left(A \otimes \mathfrak{g} \otimes \mathfrak{m}_{\mathrm{A}}\right)=\exp \left(A^{0} \otimes \mathfrak{g} \otimes \mathfrak{m}_{\mathrm{A}}\right)
$$


acts naturally on $\mathscr{F}\left(A \otimes \mathfrak{g} \otimes \mathrm{m}_{\mathrm{A}}\right)$. If $A$ is an augmented CDGA, we have that $\mathscr{F}\left(\widetilde{A} \otimes \mathfrak{g} \otimes \mathrm{m}_{\mathrm{A}}\right)=$ $\mathscr{F}\left(A \otimes \mathfrak{g} \otimes \mathrm{m}_{\mathrm{A}}\right)$ and $\mathscr{G}\left(\widetilde{A} \otimes \mathfrak{g} \otimes \mathrm{m}_{\mathrm{A}}\right) \subseteq \mathscr{G}\left(A \otimes \mathfrak{g} \otimes \mathrm{m}_{\mathrm{A}}\right)$, with the augmented gauge group acting by restriction. In the particular case when $A$ is connected, the augmented gauge group is trivial, and we obtain a natural identification,

$$
\mathscr{F}\left(A, \mathfrak{g} \otimes \mathrm{m}_{\mathrm{A}}\right)=\operatorname{Def}_{\widetilde{A} \otimes \mathfrak{g}}(\mathrm{A}) .
$$

If both $A^{1}$ and $\mathfrak{g}$ are finite-dimensional, then the set $\mathscr{F}(A, \mathfrak{g})$ has a natural structure of affine variety, which we shall call the $\mathfrak{g}$-variety of flat connections on the CDGA $A$.

Now let $(X, x)$ be a pointed space with fundamental group $\pi$, and let $G$ be a linear algebraic group over $\mathbb{k}=\mathbb{R}$ or $\mathbb{C}$, with Lie algebra $\mathfrak{g}$. The monodromy construction from $[9, \S 6.3]$ gives a map

$$
\text { mon: } \mathscr{F}(\Omega(X, \mathbb{k}), \mathfrak{g}) \longrightarrow \operatorname{Hom}(\pi, G)
$$

which extends the classical monodromy map for smooth manifolds, and has nice naturality properties. Furthermore, for each local Artin $\mathbb{k}$-algebra $A$, we have a natural monodromy map

$$
\text { mon: } \mathscr{F}\left(\Omega(X, \mathbb{k}), \mathfrak{g} \otimes \mathfrak{m}_{\mathrm{A}}\right) \longrightarrow \operatorname{Hom}\left(\pi, \exp \left(\mathfrak{g} \otimes \mathrm{m}_{\mathrm{A}}\right)\right) \text {. }
$$

The equivariance property of the monodromy map for smooth manifolds described in $[15,(5-8)]$ can be extended to arbitrary topological spaces, as follows.

Lemma 4.7. For any gauge equivalence $a \in \mathscr{G}\left(\Omega(X, \mathbb{k}) \otimes \mathfrak{g} \otimes \mathrm{m}_{A}\right)$, we have a commuting diagram,

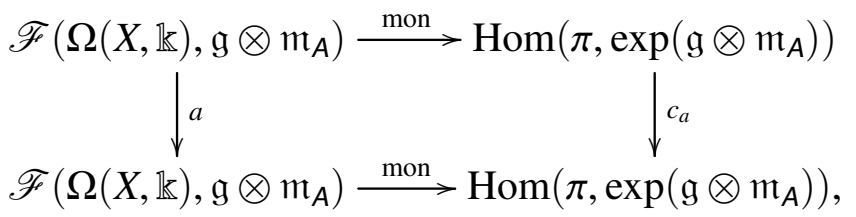

where $c_{a}$ stands for the conjugation action by $-(\varepsilon \otimes \mathrm{id})(a)$ and $\varepsilon \otimes \mathrm{id}: \Omega^{0}(X, \mathbb{k}) \otimes \mathfrak{g} \otimes$ $\mathrm{m}_{A} \rightarrow \mathfrak{g} \otimes \mathrm{m}_{A}$ is given by the augmentation $\varepsilon$ of $\Omega(X, \mathbb{k})$ corresponding to the basepoint $x$. Consequently, the monodromy map factors through the action of the augmented gauge group.

We will repeatedly work under the assumptions of Theorem B from [9]. Namely, we fix an integer $q \geqslant 1$, and we let $X$ be a pointed, $q$-finite space with fundamental group $\pi$. Next, we assume there is a $q$-finite CDGA $A$ such that $\Omega(X, \mathbb{k}) \simeq_{q} A$ in CDGA. Finally, we let $G$ be a linear algebraic group over $\mathbb{k}=\mathbb{R}$ or $\mathbb{C}$, with Lie algebra $\mathfrak{g}$.

Now let $\rho_{1}: \mathscr{N} \rightarrow \Omega(X, \mathbb{K})$ be a ' $\pi_{1}$-adapted' 1-minimal model map, as in [9, $\left.\S 6.4\right]$. By minimal model theory of CDGAs, we may extend $\rho_{1}$ to a $q$-minimal model map, $\rho_{q}: \mathscr{M}_{q} \rightarrow$ $\Omega(X, \mathbb{k})$. By Lemma 3.3, we may find a zig-zag of $q$-equivalences in ACDGA of the form

$$
S: \Omega(X, \mathbb{k}) \stackrel{\rho_{q}}{\longleftarrow} \mathscr{M}_{q} \stackrel{\bar{\rho}_{q}}{\longrightarrow} A
$$


which fits into the basic setup from [9, §7.2]. We will call such zig-zag special.

The next result is a topological analog of Theorem 6.8 from [15], proved only for smooth manifolds.

Theorem 4.8. Let $X$ be a 1-finite space. Then the natural map

$$
\text { mon: } \mathscr{F}\left(\Omega(X, \mathbb{k}) \otimes \mathfrak{g} \otimes \mathfrak{m}_{A}\right) / \mathscr{G}\left(\widetilde{\Omega}(X, \mathbb{K}) \otimes \mathfrak{g} \otimes \mathfrak{m}_{A}\right) \longrightarrow \operatorname{Hom}\left(\pi, \exp \left(\mathfrak{g} \otimes \mathfrak{m}_{A}\right)\right)
$$

from Lemma 4.7 is a bijection, for all local Artin $\mathbb{k}$-algebras $A$.

Proof. Let $\rho_{1}: \mathscr{N} \rightarrow \Omega(X, \mathbb{k})$ be a $\pi_{1}$-adapted 1-minimal model map. By [9, Prop. 6.16], the composite

$$
\mathscr{F}\left(\mathscr{N} \otimes \mathfrak{g} \otimes \mathfrak{m}_{\mathrm{A}}\right) \stackrel{\rho_{1} \otimes \mathrm{id}}{\longrightarrow} \mathscr{F}\left(\Omega(X, \mathbb{K}) \otimes \mathfrak{g} \otimes \mathfrak{m}_{\mathrm{A}}\right) \stackrel{\text { mon }}{\longrightarrow} \operatorname{Hom}\left(\pi, \exp \left(\mathfrak{g} \otimes \mathfrak{m}_{\mathrm{A}}\right)\right)
$$

is a bijection. Since $\mathscr{N}$ is connected, formula (4.9) allows us to replace $\mathscr{F}\left(\mathscr{N} \otimes \mathfrak{g} \otimes \mathrm{m}_{\mathrm{A}}\right)$

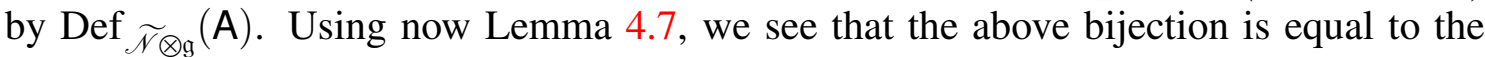
composite

$$
\operatorname{Def}_{\widetilde{N} \otimes \mathfrak{g}}(A) \stackrel{\tilde{\rho}_{1} \otimes \mathrm{id}}{\longrightarrow} \operatorname{Def}_{\widetilde{\Omega}(X, \mathbb{k}) \otimes \mathfrak{g}}(\mathrm{A}) \stackrel{\text { mon }}{\longrightarrow} \operatorname{Hom}\left(\pi, \exp \left(\mathfrak{g} \otimes \mathrm{m}_{\mathrm{A}}\right)\right)
$$

Finally, it follows from Theorem 4.1 and Remark 4.4 that the map $\tilde{\rho}_{1} \otimes$ id is also a bijection, and this completes the proof.

Assume again that the hypotheses of Theorem B from [9] are satisfied. Let $Z$ be a zigzag of $q$-equivalences in ACDGA as in (4.6), connecting $A_{0}=\Omega(X, \mathbb{k})$ to $A_{\ell}=A$. Using Theorem 4.8 and formula (4.9), we may then define a natural bijection

$$
\alpha_{Z}:=\operatorname{mon} \circ \beta_{Z}: \mathscr{F}\left(A, \mathfrak{g} \otimes \mathrm{m}_{\mathrm{A}}\right) \stackrel{\simeq}{\longrightarrow} \operatorname{Hom}\left(\pi, \exp \left(\mathfrak{g} \otimes \mathrm{m}_{\mathrm{A}}\right)\right) .
$$

Corollary 4.9. For any zig-zag $Z$ as above, there is a special zig-zag $S$ such that $\alpha_{Z}=\alpha_{S}$.

Proof. Let $\rho_{q}: \mathscr{M}_{q} \rightarrow \Omega(X, \mathbb{k})$ be a $q$-minimal model map extending a $\pi_{1}$-adapted 1minimal model map $\rho_{1}: \mathscr{N} \rightarrow \Omega(X, \mathbb{K})$. By Proposition 4.6, there is a special zig-zag $S$ as in diagram (4.12) such that $\beta_{Z}=\beta_{S}$. The claim follows.

\section{COHOMOLOGY JUMP LOCI AND NATURALITY PROPERTIES}

We now define two types of cohomology jump loci (one for spaces and the other for CDGAs), and study some of the naturality properties these algebraic varieties enjoy. 
5.1. Embedded cohomology jump loci. Let $(X, x)$ be a pointed, path-connected space. Set $\pi=\pi_{1}(X, X)$. For a $\mathbb{k}$-linear algebraic group $G$, the set $\operatorname{Hom}(\pi, G)$ is a parameter space for finite-dimensional local systems on $X$ of type $G$. When the space $X$ is 1-finite (or, equivalently, when the group $\pi$ is finitely generated), this parameter space is an affine $\mathbb{k}$-variety. When $\mathbb{k}=\mathbb{R}$ or $\mathbb{C}$, we let $\operatorname{Hom}(\pi, G)_{(1)}$ be the analytic germ at 1 of this variety, and we denote by $R=R(\pi, G)$ the analytic local algebra of this germ.

Given a CDGA $A^{\bullet}$ and a Lie algebra $\mathfrak{g}$, let $\mathscr{F}(A, \mathfrak{g})$ be the set of $\mathfrak{g}$-valued flat connections on $A$. When both $A^{1}$ and $\mathfrak{g}$ are finite-dimensional, this set is an affine variety. We shall denote by $\bar{R}=\bar{R}(A, \mathfrak{g})$ the analytic local algebra of the germ $\mathscr{F}(A, \mathfrak{g})_{(0)}$. Assume now that both $X$ and $A$ are 1-finite, and that $\Omega_{\mathbb{k}}(X) \simeq_{1} A$ as CDGAs. Letting $\mathfrak{g}$ be the Lie algebra of $G$, it then follows from [9, Prop. 7.6] that the local algebras $R$ and $\bar{R}$ are isomorphic.

Given a representation $\tau: \pi \rightarrow \mathrm{GL}(V)$, we let $V_{\tau}$ denote the local system on $X$ associated to $\tau$, that is, the left $\pi$-module $V$ defined by $g \cdot v=\tau(g) v$. Furthermore, we let $H^{\bullet}\left(X, V_{\tau}\right)$ be the twisted cohomology of $X$ with coefficients in this local system, see e.g. [35].

Definition 5.1. The characteristic varieties of the space $X$ in degree $i \geqslant 0$ and depth $r \geqslant 0$ with respect to a representation $\iota: G \rightarrow \mathrm{GL}(V)$ are the sets

$$
\mathscr{V}_{r}^{i}(X, \iota)=\left\{\rho \in \operatorname{Hom}(\pi, G) \mid \operatorname{dim}_{\mathbb{k}} H^{i}\left(X, V_{\iota \circ \rho}\right) \geqslant r\right\} .
$$

For each $i \geqslant 0$, the sequence $\left\{\mathscr{V}_{r}^{i}(X, \iota)\right\}_{r \geqslant 0}$ is a descending filtration of $\operatorname{Hom}(\pi, G)=$ $\mathscr{V}_{0}^{i}(X, \iota)$. In the rank 1 case, i.e., when $\iota$ is the canonical identification $\mathbb{k}^{\times} \rightarrow \mathrm{GL}_{1}(\mathbb{k})$, we will drop the map $\iota$ from the notation, and simply write $\mathscr{V}_{r}^{i}(X)$. When $X=K(\pi, 1)$ is a classifying space for the group $\pi$, we will denote the corresponding characteristic varieties by $\mathscr{V}_{r}^{i}(\pi, \iota)$.

We will refer to the pairs

$$
\left(\operatorname{Hom}(\pi, G), \mathscr{V}_{r}^{i}(X, \iota)\right)
$$

as the (global) embedded jump loci of $X$ with respect to $\iota$. Clearly, such pairs depend only on the homotopy type of $X$ and on the representation $\iota$. If $\iota$ is a rational representation and $X$ is a $q$-finite space for some $q \geqslant 1$, then the sets $\mathscr{V}_{r}^{i}(X, \iota)$ are closed subvarieties of the representation variety $\operatorname{Hom}(\pi, G)$, for all $i \leqslant q$ and $r \geqslant 0$; see [9, 4].

5.2. Infinitesimal cohomology jump loci. To define the infinitesimal counterpart of these loci, we start with a CDGA $A^{\bullet}$, a Lie algebra $\mathfrak{g}$, and a representation $\theta: \mathfrak{g} \rightarrow \mathfrak{g l}(V)$. For each flat connection $\omega \in \mathscr{F}(A, \mathfrak{g})$, we turn the tensor product $A \otimes V$ into a cochain complex,

$$
\left(A \otimes V, d_{\omega}\right): A^{0} \otimes V \stackrel{d_{\omega}}{\longrightarrow} A^{1} \otimes V \stackrel{d_{\omega}}{\longrightarrow} A^{2} \otimes V \stackrel{d_{\omega}}{\longrightarrow} \cdots,
$$

using as differential the covariant derivative $d_{\omega}=d \otimes \mathrm{id}_{V}+\operatorname{ad}_{\omega}$. Here, if $\omega=\sum_{i} a_{i} \otimes g_{i}$, with $a_{i} \in A^{1}$ and $g_{i} \in \mathfrak{g}$, then $\operatorname{ad}_{\omega}(a \otimes v)=\sum_{i} a_{i} a \otimes \theta\left(g_{i}\right)(v)$, for all $a \in A$ and $v \in V$. It is readily checked that the flatness condition on $\omega$ insures that $d_{\omega}^{2}=0$, see [9]. 
Definition 5.2. The resonance varieties of the CDGA $A^{\bullet}$ in degree $i \geqslant 0$ and depth $r \geqslant 0$ with respect to a representation $\theta: \mathfrak{g} \rightarrow \mathfrak{g l}(V)$ are the sets

$$
\mathscr{R}_{r}^{i}(A, \theta)=\left\{\omega \in \mathscr{F}(A, \mathfrak{g}) \mid \operatorname{dim}_{\mathbb{K}} H^{i}\left(A \otimes V, d_{\omega}\right) \geqslant r\right\} .
$$

For each $i \geqslant 0$, the sequence $\left\{\mathscr{R}_{r}^{i}(A, \theta)\right\}_{r \geqslant 0}$ is a descending filtration of $\mathscr{F}(A, \mathfrak{g})=$ $\mathscr{R}_{0}^{i}(A, \theta)$. In the rank one case, i.e., the case when $\theta$ is the canonical identification $\mathbb{k} \rightarrow$ $\mathfrak{g l}_{1}(\mathbb{k})$, we will simply write $\mathscr{R}_{r}^{i}(A)$ for the corresponding sets.

We will refer to the pairs

$$
\left(\mathscr{F}(A, \mathfrak{g}), \mathscr{R}_{r}^{i}(A, \theta)\right)
$$

as the (global) infinitesimal embedded jump loci of $A$ with respect to $\theta$. If $A$ is $q$-finite for some $q \geqslant 1$, and both $\mathfrak{g}$ and $V$ are finite-dimensional, the sets $\mathscr{R}_{r}^{i}(A, \theta)$ are closed subvarieties of $\mathscr{F}(A, \mathfrak{g})$, for all $i \leqslant q$ and $r \geqslant 0$; see [9, 4].

Assume now that both the space $X$ and the CDGA $A^{\bullet}$ are $q$-finite, for some $q \geqslant 1$, and that $\Omega_{\mathbb{k}}(X) \simeq_{q} A$ as CDGAs. Let $\iota: G \rightarrow \mathrm{GL}(V)$ be a rational representation, and let $\theta: \mathfrak{g} \rightarrow \mathfrak{g l}(V)$ be its tangential representation. As shown in [9, Thm. B], there is then an analytic isomorphism $\mathscr{F}(A, \mathfrak{g})_{(0)} \stackrel{\simeq}{\longrightarrow} \operatorname{Hom}(\pi, G)_{(1)}$ restricting to isomorphisms $\mathscr{R}_{r}^{i}(A, \theta)_{(0)} \stackrel{\simeq}{\longrightarrow} \mathscr{V}_{r}^{i}(X, \iota)_{(1)}$ between the reduced analytic germs of the corresponding jump loci, for all $i \leqslant q$ and $r \geqslant 0$.

We aim in this section at also taking into account in this setting of continuous maps between pointed spaces and of augmented maps between their $q$-models. We start with a preliminary observation, which follows directly from the definitions. Namely, for all $i \leqslant q$ and $r \geqslant 0$

$$
1 \in \mathscr{V}_{r}^{i}(X, \iota) \Leftrightarrow 0 \in \mathscr{R}_{r}^{i}(A, \theta) \Leftrightarrow b_{i} \cdot \operatorname{dim}(V) \geqslant r,
$$

where $b_{i}=b_{i}(X)=b_{i}(A)$ denotes the $i$-th (untwisted) Betti number.

5.3. Naturality properties of representation varieties. As mentioned previously, both ambient spaces for jump $\operatorname{loci}, \operatorname{Hom}(\pi, G)$ and $\mathscr{F}(A, \mathfrak{g})$, are bifunctorial. On the other hand, for continuous and CDGA maps, naturality of (global) jump loci requires certain connectivity hypotheses. To begin, we only assume the minimally required connectivity and finiteness conditions.

Lemma 5.3. Let $f:(X, x) \rightarrow\left(X^{\prime}, x^{\prime}\right)$ be a 0 -connected, pointed map, and let $f_{\sharp}$ be the induced homomorphism on fundamental groups. Assume that $X$ is 1-finite. Then, for every linear algebraic group $G$, the morphism induced by $f_{\sharp}$ on representation varieties,

$$
f_{\sharp}^{!}: \operatorname{Hom}\left(\pi_{1}\left(X^{\prime}\right), G\right) \longrightarrow \operatorname{Hom}\left(\pi_{1}(X), G\right),
$$

is an isomorphism onto a closed subvariety.

Proof. Our 0-connectivity assumption on $f$ means that the homomorphism $f_{\sharp}$ is surjective. Our 1-finiteness assumption on $X$, then, implies that both fundamental groups are finitely 
generated. Let us present $\pi_{1}(X)$ as the quotient $F_{m} / R$ of a free group on $m$ generators, and then use the presentation for $\pi_{1}\left(X^{\prime}\right)$ induced by $f_{\sharp}$.

By construction, the representation variety $\operatorname{Hom}\left(\pi_{1}(X), G\right)$ is the closed subvariety of $G^{m}$ defined by the equations given by the relators in $R$. The variety $\operatorname{Hom}\left(\pi_{1}\left(X^{\prime}\right), G\right)$ sits also in $G^{m}$, with the same defining equations as $\operatorname{Hom}\left(\pi_{1}(X), G\right)$, plus the equations coming from the lifts to $F_{m}$ of the elements of $\operatorname{ker}\left(f_{\sharp}\right)$. The claim readily follows.

5.4. Holonomy Lie algebras. Before proceeding, let us recall from [22, §4] the construction of the holonomy Lie algebra $\mathfrak{h}(A)$ of a 1-finite CDGA $(A, d)$. Set $A_{i}=\left(A^{i}\right)^{*}$, and let $\mathbb{L}\left(A_{1}\right)$ be the free Lie algebra on the dual vector space $A_{1}$. We then define

$$
\mathfrak{h}(A):=\mathbb{L}\left(A_{1}\right) / \operatorname{ideal}\left(\operatorname{im}\left(d^{*}+\cup^{*}\right)\right),
$$

where $d^{*}: A_{2} \rightarrow A_{1}=\mathbb{L}^{1}\left(A_{1}\right)$ and $\cup^{*}: A_{2} \rightarrow A_{1} \wedge A_{1}=\mathbb{L}^{2}\left(A_{1}\right)$ are the maps dual to the differential and the multiplication map in $A$, respectively. This construction is functorial: if $\psi: A^{\prime} \rightarrow A$ is a morphism of 1-finite CDGAs, then the linear map $\psi_{1}=\left(\psi^{1}\right)^{*}: A_{1} \rightarrow$ $A_{1}^{\prime}$ extends to a Lie algebra morphism $\mathbb{L}\left(\psi_{1}\right): \mathbb{L}\left(A_{1}\right) \rightarrow \mathbb{L}\left(A_{1}^{\prime}\right)$, which in turn induces a Lie algebra morphism $\mathfrak{h}(\psi): \mathfrak{h}(A) \rightarrow \mathfrak{h}\left(A^{\prime}\right)$. Finally, as shown in [22, Prop. 4.5], the canonical isomorphism $A^{1} \otimes \mathfrak{g} \stackrel{\simeq}{\operatorname{Hom}}\left(A_{1}, \mathfrak{g}\right)$ restricts to an identification $\mathscr{F}(A, \mathfrak{g}) \cong$ $\operatorname{Hom}_{\text {Lie }}(\mathfrak{h}(A), \mathfrak{g})$.

Lemma 5.4. Let $\psi: A^{\prime} \rightarrow A$ be a 0 -connected CDGA map. Assume that $A$ is 1-finite. Then, for every finite dimensional Lie algebra $\mathfrak{g}$, the morphism

$$
\psi \otimes \mathrm{id}: \mathscr{F}\left(A^{\prime}, \mathfrak{g}\right) \longrightarrow \mathscr{F}(A, \mathfrak{g})
$$

is an isomorphism onto a closed subvariety.

Proof. Our 0-connectivity assumption on $\psi$ means that both $A$ and $A^{\prime}$ are connected CDGAs, and that $\psi$ is injective in degree 1. Our 1-finiteness assumption on $A$, then, implies that $A^{\prime}$ is also 1-finite. Furthermore, the injectivity of $\psi^{1}$ also implies that the map $\mathfrak{h}(\psi): \mathfrak{h}(A) \rightarrow \mathfrak{h}\left(A^{\prime}\right)$ is surjective.

Using the above discussion, we may replace the affine map $\psi \otimes \mathrm{id}: \mathscr{F}\left(A^{\prime}, \mathfrak{g}\right) \rightarrow \mathscr{F}(A, \mathfrak{g})$ between spaces of flat connections by the induced map

$$
\mathfrak{h}(\psi)^{!}: \operatorname{Hom}_{\mathrm{Lie}}\left(\mathfrak{h}\left(A^{\prime}\right), \mathfrak{g}\right) \longrightarrow \operatorname{Hom}_{\mathrm{Lie}}(\mathfrak{h}(A), \mathfrak{g})
$$

between representation varieties of Lie algebras. The desired conclusion follows by the same argument as in Lemma 5.3, with groups replaced by Lie algebras.

As we saw in the above proof, the 0-connectivity of the CDGA map $\psi$ implies the surjectivity of the Lie algebra map $\mathfrak{h}(\psi)$. The next example shows that the latter property is strictly weaker than the former. Nevertheless, we chose to state the lemma the way we did, since higher connectivity properties for CDGA maps will be needed later on. 
Example 5.5. Let $A$ be the cohomology ring of $S^{1} \vee S^{1}$, with trivial product and differential. Plainly, the holonomy Lie algebra $\mathfrak{h}=\mathfrak{h}(A)$ is the free Lie algebra on 2 generators. Let $\mathfrak{h} / \Gamma_{3}(\mathfrak{h})$ be the third nilpotent quotient of $\mathfrak{h}$, and let $A^{\prime}$ be the cochain CDGA of this nilpotent Lie algebra. Denote by $\psi: A^{\prime 1} \rightarrow A^{1}$ the dual of the composite $A_{1} \rightarrow \mathfrak{h} \rightarrow \mathfrak{h} / \Gamma_{3} \mathfrak{h}$. It is not hard to check that $\psi$ extends to a morphism $\psi: A^{\prime} \rightarrow A$ between finite CDGAs, with the property that $\mathfrak{h}(\psi)$ is surjective. On the other hand, the map $\psi$ is not 0 -connected, as can be seen by inspecting dimensions in degree 1 .

5.5. Naturality properties of jump loci. We now turn to the naturality properties of embedded cohomology jump loci.

Lemma 5.6. Let $f:(X, x) \rightarrow\left(X^{\prime}, x^{\prime}\right)$ be a q-connected, pointed map, and let $f_{\sharp}$ be the induced homomorphism on fundamental groups. Let $\iota: G \rightarrow \mathrm{GL}(V)$ be a representation. Then the natural map

$$
H^{\bullet}(f): H^{\bullet}\left(X^{\prime}, V_{\iota \circ \rho^{\prime}}\right) \longrightarrow H^{\bullet}\left(X, V_{\iota \circ \rho}\right),
$$

where $\rho=f_{\sharp}^{!}\left(\rho^{\prime}\right)$ for $\rho^{\prime} \in \operatorname{Hom}\left(\pi_{1}\left(X^{\prime}\right), G\right)$, is q-connected.

Proof. Without loss of generality, we may assume that $G=\mathrm{GL}(V)$ and $\iota$ is the identity map. Using standard CW-approximation results from homotopy theory, as recounted for instance in [35, Ch. V], we may replace $f$, up to homotopy, by the inclusion of a $\mathrm{CW}$ subcomplex $X$ into a CW-complex $X^{\prime}$. Since $f$ is assumed to be $q$-connected, $X^{\prime}$ may be obtained by attaching cells of dimension at least $q+2$ to $X$.

Using the long exact sequence in cohomology for the pair $\left(X^{\prime}, X\right)$, we see that our claim is equivalent to the vanishing of the twisted cohomology groups $H^{i}\left(X^{\prime}, X ; V\right)$ for $i \leqslant q+1$, for an arbitrary local system $V$ on $X^{\prime}$. Denote by $\left\{X_{n}^{\prime}\right\}$ the relative skeletal filtration of $X^{\prime}$. It is well-known that $H^{\bullet}\left(X^{\prime}, X ; V\right)$ can be computed as the cohomology of the cellular twisted cochain complex, whose degree $n$ term is $H^{n}\left(X_{n}^{\prime}, X_{n-1}^{\prime} ; V\right)$, see e.g. [35, Ch. VI]. On the other hand, $X_{n}^{\prime}=X_{n-1}^{\prime}=X$ for $n \leqslant q+1$, and this completes the proof.

Lemma 5.7. Let $\psi: A^{\prime} \rightarrow A$ be a q-connected map in CDGA, and let $\theta: \mathfrak{g} \rightarrow \mathfrak{g l}(V)$ be a Lie algebra representation. Then the natural map

$$
H^{\bullet}(\psi): H^{\bullet}\left(A^{\prime} \otimes V, d_{\omega^{\prime}}\right) \longrightarrow H^{\bullet}\left(A \otimes V, d_{\omega}\right),
$$

where $\omega=(\psi \otimes \mathrm{id})\left(\omega^{\prime}\right)$ for $\omega^{\prime} \in \mathscr{F}\left(A^{\prime}, \mathfrak{g}\right)$ is q-connected.

Proof. Without loss of generality, we may assume that $\mathfrak{g}=\mathfrak{g l}(V)$ and $\theta$ is the identity map. Since $\psi$ is $q$-connected, the cochain map $\psi \otimes \mathrm{id}:\left(A^{\prime} \otimes V, d_{\omega^{\prime}}\right) \rightarrow\left(A \otimes V, d_{\omega}\right)$ is again $q$-connected. The claim follows from Lemma 2.6 in [22] and its proof.

Corollary 5.8. Let $f: X \rightarrow X^{\prime}$ be a $(q-1)$-connected map between q-finite pointed spaces, for some $q \geqslant 1$, and let $\iota: G \rightarrow \mathrm{GL}(V)$ be a rational representation. Then the induced morphism

$$
f_{\sharp}^{!}: \operatorname{Hom}\left(\pi_{1}\left(X^{\prime}\right), G\right) \longrightarrow \operatorname{Hom}\left(\pi_{1}(X), G\right)
$$


is a closed embedding which induces isomorphisms $\mathscr{V}_{r}^{i}\left(X^{\prime}, \iota\right) \rightarrow \mathscr{V}_{r}^{i}(X, \iota) \cap \operatorname{Hom}\left(\pi_{1}\left(X^{\prime}\right), G\right)$ for all $i<q$ and $r \geqslant 0$, and embeddings $\mathscr{V}_{r}^{q}\left(X^{\prime}, \iota\right) \rightarrow \mathscr{V}_{r}^{q}(X, \iota)$ for all $r \geqslant 0$.

Proof. The fact that $f_{\sharp}^{!}$is a closed embedding follows from Lemma 5.3. The other assertions are immediate consequences of Lemma 5.6.

If $f: \pi \rightarrow \pi^{\prime}$ is an epimorphism between finitely generated groups, the case $q=1$ from Corollary 5.8 implies that the morphism $f^{!}: \operatorname{Hom}\left(\pi^{\prime}, \mathbb{k}^{\times}\right) \rightarrow \operatorname{Hom}\left(\pi, \mathbb{R}^{\times}\right)$sends $\mathscr{V}_{1}^{1}\left(\pi^{\prime}\right)$ into $\mathscr{V}_{1}^{1}(\pi)$. Without the 0 -connectivity (i.e., surjectivity) assumption on $f$, the conclusion may fail, as illustrated in the following simple example.

Example 5.9. Let $f: \mathbb{Z} \rightarrow F_{2}=\langle x, y\rangle$ be the inclusion sending 1 to $x$. Then $f^{!}:\left(\mathbb{R}^{\times}\right)^{2} \rightarrow$ $\mathbb{K}^{\times}$is the projection onto the first factor. On the other hand, $\mathscr{V}_{1}^{1}\left(F_{2}\right)=\left(\mathbb{k}^{\times}\right)^{2}$, whereas $\mathscr{V}_{1}^{1}(\mathbb{Z})=\{1\}$.

Corollary 5.10. Let $\psi: A^{\prime} \rightarrow A$ be a $(q-1)$-connected map between q-finite CDGAs for some $q \geqslant 1$, and let $\theta: \mathfrak{g} \rightarrow \mathfrak{g l}(V)$ be a Lie algebra representation, with $\mathfrak{g}$ and $V$ finite-dimensional. Then the natural morphism

$$
\psi \otimes \mathrm{id}: \mathscr{F}\left(A^{\prime}, \mathfrak{g}\right) \longrightarrow \mathscr{F}(A, \mathfrak{g})
$$

is a closed embedding which induces isomorphisms $\mathscr{R}_{r}^{i}\left(A^{\prime}, \theta\right) \rightarrow \mathscr{R}_{r}^{i}(A, \theta) \cap \mathscr{F}\left(A^{\prime}, \mathfrak{g}\right)$ for all $i<q$ and $r \geqslant 0$, and embeddings $\mathscr{R}_{r}^{q}\left(A^{\prime}, \theta\right) \rightarrow \mathscr{R}_{r}^{q}(A, \theta)$ for all $r \geqslant 0$.

Proof. The fact that $\psi \otimes$ id is a closed embedding follows from Lemma 5.4. The other assertions are immediate consequences of Lemma 5.7.

Corollary 5.11. Let $X$ be a pointed space with fundamental group $\pi$, let $f: X \rightarrow K:=$ $K(\pi, 1)$ be a classifying map, and let $\iota: G \rightarrow \mathrm{GL}(V)$ be a representation. Then the induced isomorphism $f_{\sharp}^{!}: \operatorname{Hom}\left(\pi_{1}(K), G\right) \rightarrow \operatorname{Hom}\left(\pi_{1}(X), G\right)$ restricts to isomorphisms $\mathscr{V}_{r}^{i}(\pi, \iota) \cong \mathscr{V}_{r}^{i}(X, \iota)$ for $i \leqslant 1$ and $r \geqslant 0$.

Proof. The map $f$ is 1-connected, and so the claim follows from Lemma 5.6.

5.6. Finite families of epimorphisms. We conclude this section with a setup that will often recur in the sequel. Let $\pi$ be a finitely generated group, and let $\left\{f: \pi \rightarrow \pi_{f}\right\}_{f \in E}$ be a finite family of epimorphisms. Let $\iota: G \rightarrow \mathrm{GL}(V)$ be a rational representation of $\mathbb{C}$-linear algebraic groups. By Corollary 5.8, the natural inclusion

$$
\operatorname{Hom}(\pi, G) \supseteq \bigcup_{f \in E} f^{!} \operatorname{Hom}\left(\pi_{f}, G\right)
$$

induces for each $i \leqslant 1$ and $r \geqslant 1$ an inclusion

$$
\mathscr{V}_{r}^{i}(\pi, \iota) \supseteq \bigcup_{f \in E} f^{!} \mathscr{V}_{r}^{i}\left(\pi_{f}, \iota\right)
$$


One of our main goals for the remainder of this paper is to delineate several large classes of groups endowed with the required finite families of epimorphisms, for which the above two inclusions hold as equalities near 1 .

\section{A NATURAL COMPARISON BETWEEN EMBEDDED JUMP LOCI}

This section is devoted to proving our main naturality result.

6.1. Functors of Artin rings. Returning now to the setup from $\S \S 4.5-4.6$, let $X$ be a pointed, 1-finite space, and assume there is a 1-finite CDGA such that $\Omega_{\mathbb{k}}(X) \simeq_{1} A$ in CDGA. Let $\pi=\pi_{1}(X)$, and let $G$ be a linear algebraic group, with Lie algebra $\mathfrak{g}$. We wish to compare the analytic germs $\operatorname{Hom}(\pi, G)_{(1)}$ and $\mathscr{F}(A, \mathfrak{g})_{(0)}$. Let $R$ and $\bar{R}$ be the respective coordinate local algebras. By Artin approximation, we may start by looking at the completions of these rings, $\widehat{R}$ and $\widehat{\bar{R}}$. Alternatively, we may analyze the corresponding functors of Artin rings,

$$
h_{\widehat{R}}(\mathrm{~A})=\operatorname{Hom}(\widehat{R}, \mathrm{~A}) \text { and } h_{\hat{\bar{R}}}(\mathrm{~A})=\operatorname{Hom}(\hat{\bar{R}}, \mathrm{~A}) \text {, }
$$

for $A$ a local Artin algebra, where Hom stands for morphisms of local algebras. We recall that $h_{\widehat{R}}(\mathrm{~A})=\operatorname{Hom}\left(\pi, \exp \left(\mathfrak{g} \otimes \mathrm{m}_{\mathrm{A}}\right)\right)$, see [15], and $h_{\overline{\bar{R}}}(\mathrm{~A})=\mathscr{F}\left(A, \mathfrak{g} \otimes \mathfrak{m}_{\mathrm{A}}\right)$, see [9].

Let $Z$ be a zig-zag of 1-equivalences in ACDGA connecting $\Omega_{\mathbb{k}}(X)$ to $A$, as in (4.6). It follows from Theorem 4.8 that the natural bijection $\alpha_{Z}$ defined in (4.13) yields an isomorphism

$$
\alpha_{Z}: \widehat{R} \longrightarrow \widehat{\bar{R}} .
$$

Assume now that $X$ and $A$ are $q$-finite, for some $q \geqslant 1$, and that $\Omega_{\mathbb{k}}(X) \simeq_{q} A$ in CDGA. As usual, let $\iota: G \rightarrow \mathrm{GL}(V)$ be a rational representation, with tangential representation $\theta: \mathfrak{g} \rightarrow \mathfrak{g l}(V)$. For each $i \leqslant q$ and $r \geqslant 0$, we denote by $I_{r}^{i} \subseteq R$ the radical of the defining ideal of the germ $\mathscr{V}_{r}^{i}(X, \iota)_{(1)}$ inside $\operatorname{Hom}(\pi, G)_{(1)}$. Similarly, we will let $\bar{I}_{r}^{i} \subseteq \bar{R}$ stand for the radical of the defining ideal of the germ $\mathscr{R}_{r}^{i}(A, \theta)_{(0)}$ inside $\mathscr{F}(A, \mathfrak{g})_{(0)}$.

Lemma 6.1. For any zig-zag $Z$ as above, the isomorphism $\alpha_{Z}: \widehat{R} \rightarrow \widehat{\bar{R}}$ from (6.2) identifies $\widehat{I_{r}^{i}}$ with $\widehat{\bar{I}}_{r}^{i}$, for all $i \leqslant q$ and $r \geqslant 0$.

Proof. In view of Corollary 4.9, we may replace the zig-zag $Z$ by a special zig-zag $S$. The claim for $S$ follows from [9, Lem. 9.9].

Now let $f: X \rightarrow X^{\prime}$ be a pointed map, let $\Phi: A^{\prime} \rightarrow A$ be a CDGA map, and assume both spaces and CDGAs are $q$-finite, for some $q \geqslant 1$. Let $\phi: R \rightarrow R^{\prime}$ be the morphism of local rings induced by the map $f_{\sharp}^{!}: \operatorname{Hom}\left(\pi^{\prime}, G\right) \rightarrow \operatorname{Hom}(\pi, G)$, and let $\bar{\phi}: \bar{R} \rightarrow \bar{R}^{\prime}$ be the morphism of local rings induced by the map $\Phi \otimes \mathrm{id}: \mathscr{F}\left(A^{\prime}, \mathfrak{g}\right) \rightarrow \mathscr{F}(A, \mathfrak{g})$. 
Suppose there is a $q$-equivalence in $\mathrm{ACDGA}_{0}$ between the maps $\Omega_{\mathrm{k}}(f): \Omega_{\mathrm{k}}\left(X^{\prime}\right) \rightarrow \Omega_{\mathbb{k}}(X)$ and $\Phi: A^{\prime} \rightarrow A$. We then obtain zig-zags $Z^{\prime}$ from $\Omega_{\mathbb{k}}\left(X^{\prime}\right)$ to $A^{\prime}$ and $Z$ from $\Omega_{\mathbb{k}}(X)$ to $A$; let $\alpha^{\prime}: \hat{R}^{\prime} \rightarrow \hat{\overline{R^{\prime}}}$ and $\alpha: \hat{R} \rightarrow \hat{\bar{R}}$ be the corresponding isomorphisms, given by (6.2).

Lemma 6.2. With the above setup, we have that $\alpha^{\prime} \circ \hat{\phi}=\hat{\bar{\phi}} \circ \alpha$.

Proof. In terms of functors of Artin rings, we have that $h_{\alpha}=\operatorname{mon} \circ \beta_{Z}$ and $h_{\alpha^{\prime}}=\operatorname{mon} \circ \beta_{Z^{\prime}}$. First we show that the following diagram commutes, for every local Artin algebra $A$.

$$
\begin{aligned}
& \mathscr{F}\left(A, \mathfrak{g} \otimes \mathfrak{m}_{\mathrm{A}}\right)=\frac{\mathscr{F}\left(\widetilde{A} \otimes \mathfrak{g} \otimes \mathfrak{m}_{\mathrm{A}}\right)}{\mathscr{G}\left(\widetilde{A} \otimes \mathfrak{g} \otimes \mathfrak{m}_{\mathrm{A}}\right)} \stackrel{\beta_{Z}}{\longrightarrow} \frac{\mathscr{F}\left(\widetilde{\Omega}(X) \otimes \mathfrak{g} \otimes \mathfrak{m}_{\mathrm{A}}\right)}{\mathscr{G}\left(\widetilde{\Omega}(X) \otimes \mathfrak{g} \otimes \mathfrak{m}_{\mathrm{A}}\right)}
\end{aligned}
$$

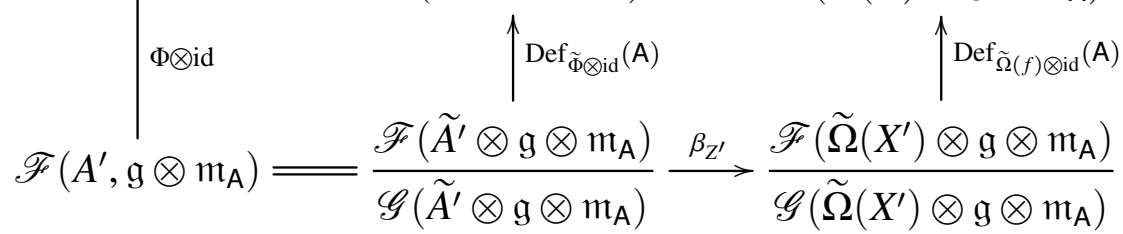

Plainly, it is enough to verify the commutativity of this diagram for an elementary $q$ equivalence in $\mathrm{ACDGA}_{0}$. In this case, $\beta_{Z}=\operatorname{Def}_{\tilde{\psi} \otimes i \mathrm{id}}(\mathrm{A})$ and $\beta_{Z^{\prime}}=\operatorname{Def}_{\tilde{\psi}^{\prime} \otimes i \mathrm{~d}}(\mathrm{~A})$, by construction. The claim now follows from Lemma 4.5 and Corollary 4.3.

Next, we show that the diagram

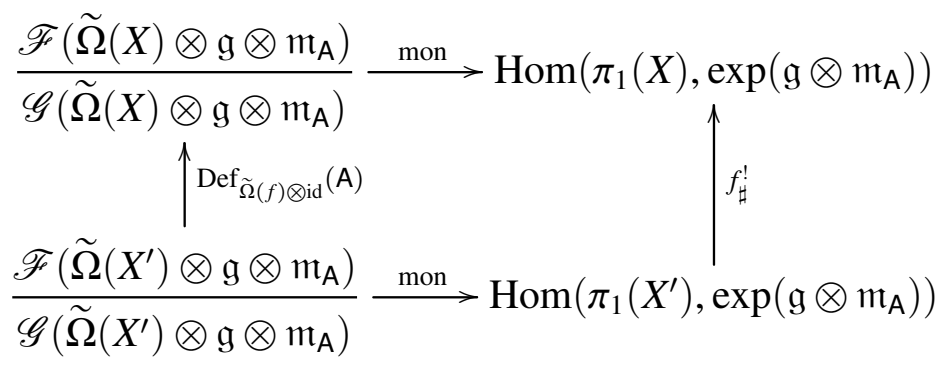

commutes, where the horizontal arrows are as in Theorem 4.8. In fact, commutativity holds even before taking quotients by the gauge actions, due to the naturality properties of the monodromy construction, as detailed in [9, §6.3].

It is now straightforward to check that the natural transformation between functors of Artin rings induced by $\hat{\bar{\phi}}$ takes the value $\Phi \otimes$ id on $A$, whereas for $\hat{\phi}$ we obtain the value $f_{\sharp}^{!}$. The commutativity of the above two diagrams now verifies the claim.

6.2. A natural comparison between embedded jump loci. We now consider a family of maps between pointed spaces, $\left\{f: X \rightarrow X_{f}\right\}_{f \in E}$, indexed by a finite set $E$, and we let $\left\{f_{\sharp}: \pi \rightarrow \pi_{f}\right\}$ be the family of induced homomorphisms on fundamental groups. We also consider a family of ACDGA maps, $\left\{\Phi_{f}: A_{f} \rightarrow A\right\}_{f \in E}$, indexed by the same set, and we will assume that $A$ and $A_{f}$ are connected CDGAs. Fix an integer $q \geqslant 1$. 
Suppose that $\Omega(f) \simeq_{q} \Phi_{f}$ in ACDGA . We then have a commuting diagram as in (3.4),

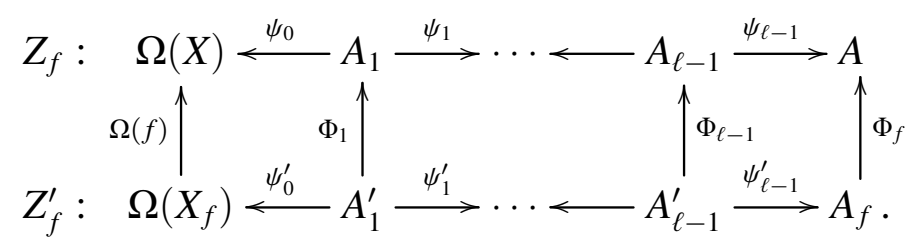

Let $\beta_{Z_{f}}$ and $\beta_{Z_{f}^{\prime}}$ be the associated natural bijections, defined as in display (4.7).

Definition 6.3. We will say that $\Omega(f) \simeq_{q} \Phi_{f}$ in $\mathrm{ACDGA}_{0}$, uniformly with respect to $f \in E$ if the bijection $\beta_{Z_{f}}$ is independent of $f$.

We are now in a position to state and prove our main naturality result. As usual, $G$ is a $\mathbb{k}$-linear algebraic group (where $\mathbb{k}=\mathbb{R}$ or $\mathbb{C}$ ), and $\mathfrak{g}$ is its Lie algebra. Furthermore, we consider a rational representation $\iota: G \rightarrow \mathrm{GL}(V)$ over $\mathbb{k}$, and we let $\theta: \mathfrak{g} \rightarrow \mathfrak{g l}(V)$ be the tangential representation.

Theorem 6.4. Suppose the following conditions hold:

(1) All the above spaces and CDGAs are q-finite.

(2) Both $f$ and $\Phi_{f}$ are $(q-1)$-connected maps, for all $f \in E$.

(3) $\Omega(f) \simeq_{q} \Phi_{f}$ in $\mathrm{ACDGA}_{0}$, uniformly with respect to $f \in E$.

Then we may find local analytic isomorphisms $a: \mathscr{F}(A, \mathfrak{g})_{(0)} \stackrel{\simeq}{\longrightarrow} \operatorname{Hom}(\pi, G)_{(1)}$ and $a_{f}: \mathscr{F}\left(A_{f}, \mathfrak{g}\right)_{(0)} \stackrel{\simeq}{\longrightarrow} \operatorname{Hom}\left(\pi_{f}, G\right)_{(1)}$ for all $f \in E$ such that the following diagram commutes, for all $f \in E$,

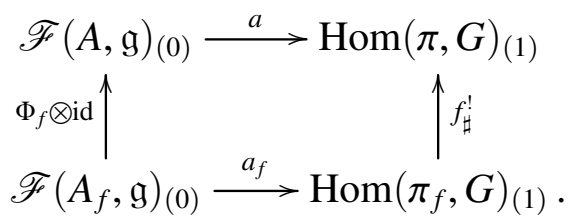

Moreover, for all $f \in E, i \leqslant q$, and $r \geqslant 0$, this construction induces a commuting diagram of (local, reduced) embedded jump loci,

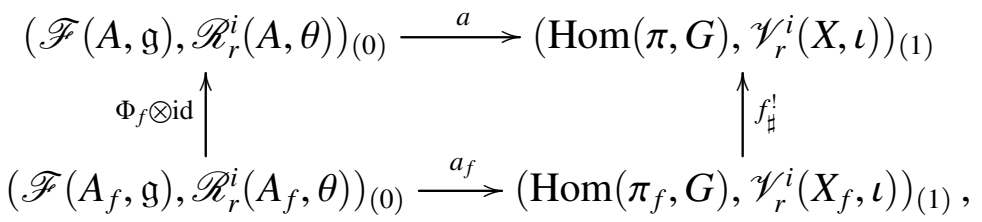

where both horizontal arrows are isomorphisms of analytic pairs.

Proof. Due to our connectivity assumptions, Corollaries 5.8 and 5.10 apply, thereby showing that both $f_{\sharp}^{!}$and $\Phi_{f} \otimes$ id respect the corresponding (global) jump loci. We will deduce all other claims from Proposition 2.3.

To begin with, we denote by $\phi_{f}: R \rightarrow R_{f}$ and $\bar{\phi}_{f}: \bar{R} \rightarrow \bar{R}_{f}$ the morphisms of analytic algebras corresponding to the local analytic maps $f_{\sharp}^{!}: \operatorname{Hom}\left(\pi_{f}, G\right)_{(1)} \rightarrow \operatorname{Hom}(\pi, G)_{(1)}$ 
and $\Phi_{f} \otimes \mathrm{id}: \mathscr{F}\left(A_{f}, \mathfrak{g}\right)_{(0)} \rightarrow \mathscr{F}(A, \mathfrak{g})_{(0)}$, respectively. To verify that both $\phi_{f}$ and $\bar{\phi}_{f}$ are epimorphisms, we may use a standard, equivalent property, namely, the injectivity of the associated natural transformation between Hom-functors, see for instance [33, III.4]. In turn, this property readily follows from the injectivity on A-points of the corresponding morphisms between affine coordinate rings, for an arbitrary commutative algebra $A$.

For representation varieties, the map on A-points is given by $f_{\sharp}^{!}: \operatorname{Hom}\left(\pi_{f}, G(\mathrm{~A})\right) \rightarrow$ $\operatorname{Hom}(\pi, G(\mathrm{~A}))$. Clearly, this map is injective, since by assumption, $f$ is 0 -connected, i.e., $f_{\sharp}$ is surjective. Likewise, for varieties of flat connections, the map on A-points is given by $\Phi_{f} \otimes \mathrm{id}: \mathscr{F}\left(A_{f} \otimes \mathfrak{g} \otimes \mathrm{A}\right) \rightarrow \mathscr{F}(A \otimes \mathfrak{g} \otimes \mathrm{A})$. Again, this map is injective, since by assumption $\Phi_{f}$ is 0-connected, i.e., injective. This shows that the first preliminary hypotheses from Proposition 2.3 are satisfied.

For $i \leqslant q$ and $r \geqslant 0$, let $I_{r}^{i} \subseteq R$ and $\bar{I}_{r}^{i} \subseteq \bar{R}$ be defining radical ideals for the reduced analytic germs $\mathscr{V}_{r}^{i}(X, \iota)_{(1)}$ and $\mathscr{R}_{r}^{i}(A, \theta)_{(0)}$, as in Lemma 6.1. Similarly, for $f \in E$, let $I_{r}^{i}(f) \subseteq R_{f}$ and $\bar{I}_{r}^{i}(f) \subseteq \bar{R}_{f}$ be defining radical ideals for $\mathscr{V}_{r}^{i}\left(X_{f}, \iota\right)_{(1)}$, and $\mathscr{R}_{r}^{i}\left(A_{f}, \theta\right)_{(0)}$. We deduce from display (5.5) that $I_{r}^{i} \neq R$ if and only if $\bar{I}_{r}^{i} \neq \bar{R}$, which happens precisely when $r \leqslant b_{i} \cdot \operatorname{dim}(V)$, where recall $b_{i}=b_{i}(X)=b_{i}(A)$. Similarly, $I_{r}^{i}(f)$ is a proper ideal if and only $\bar{I}_{r}^{i}(f)$ is a proper ideal.

Note that $I_{r}^{i}=R$ is equivalent to $\mathscr{V}_{r}^{i}(X, \iota)_{(1)}=\varnothing$, and similarly for $I_{r}^{i}(f)$. By Corollary 5.8 , if $\mathscr{V}_{r}^{i}(X, \iota)_{(1)}$ is empty, then $\mathscr{V}_{r}^{i}\left(X_{f}, \iota\right)_{(1)}$ is also empty. Consequently, if $I_{r}^{i}$ is nonproper, then $I_{r}^{i}(f)$ is also non-proper. Likewise, Corollary 5.10 implies the following: if $\bar{I}_{r}^{i}$ is non-proper, then $\bar{I}_{r}^{i}(f)$ is also non-proper.

The pairs $(i, r)$ with $0 \leqslant i \leqslant q$ and $0 \leqslant r \leqslant b_{i} \cdot \operatorname{dim}(V)$ form a finite set, which we will denote by $F$. Plainly, we need to verify the second claim of the theorem only for the pairs $(i, r) \in F$ and the maps $f \in E$ for which the ideal $I_{r}^{i}(f)$ is proper.

By assumption (3), $\Omega(f) \simeq_{q} \Phi_{f}$ in $\mathrm{ACDGA}_{0}$, uniformly with respect to $f \in E$. In particular, we have a zig-zag $Z_{f}$ of $q$-equivalences from $\Omega(X)$ to $A$ and a zig-zag $Z_{f}^{\prime}$ from $\Omega\left(X_{f}\right)$ to $A_{f}$ for each $f \in E$, as in diagram (6.5). Let

$$
\alpha_{f}:=\operatorname{mon} \circ \beta_{Z_{f}^{\prime}}: \widehat{R}_{f} \cong \widehat{\bar{R}}_{f}
$$

be the isomorphism from (6.2). By our uniformity assumption, the isomorphisms mon $\circ \beta_{Z_{f}}$

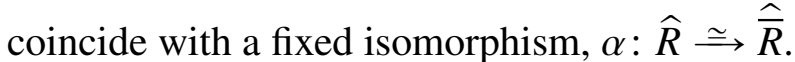

It follows from Lemma 6.1 that the isomorphism $\alpha_{f}$ identifies the ideal $\widehat{I}_{r}^{i}(f)$ with $\widehat{\bar{I}}_{r}^{i}(f)$, for all $i \leqslant q$ and $r \geqslant 0$, and for all $f \in E$. Likewise, the isomorphism $\alpha$ identifies the ideal $\widehat{I}_{r}^{i}$ with $\widehat{\bar{I}}_{r}^{i}$, for all $i \leqslant q$ and $r \geqslant 0$. Finally, assumption (4) from Proposition 2.3 follows from Lemma 6.2.

The desired conclusions follow from Proposition 2.3, applied to the above ideals. 
6.3. Naturality with respect to a single map. For a one-element family $E=\{f\}$, the uniform equivalence property from Definition 6.3 reduces to $\Omega(f) \simeq_{q} \Phi_{f}$ in $\mathrm{ACDGA}_{0}$. We thus have the following immediate corollary to Theorem 6.4.

Corollary 6.5. Let $f: X \rightarrow X^{\prime}$ be a continuous, $(q-1)$-connected map between $q$-finite, pointed spaces, for some $q \geqslant 1$. Suppose $\Phi: A^{\prime} \rightarrow A$ is a $(q-1)$-connected CDGA map between q-finite CDGAs such that $\Omega(f) \simeq_{q} \Phi$ in $\mathrm{ACDGA}_{0}$. We then may find local analytic isomorphisms $a$ and $a^{\prime}$ which fit into the diagram

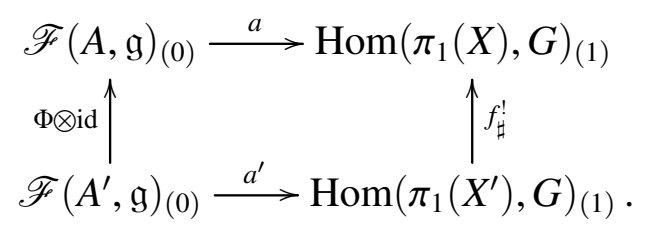

Furthermore, for all $i \leqslant q$, and $r \geqslant 0$, this construction induces a commuting diagram of (local, reduced) embedded jump loci,

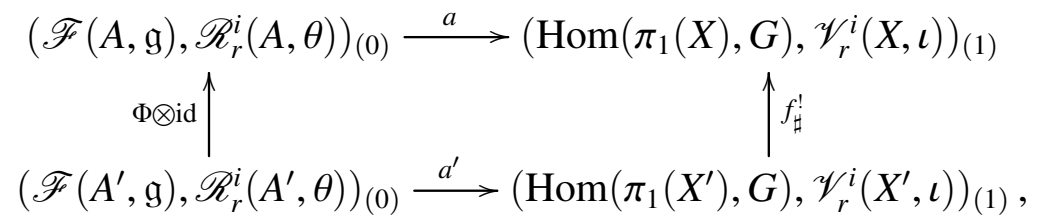

where both horizontal arrows are isomorphisms of analytic pairs.

Here is a situation where this type of property holds.

Lemma 6.6. Let $f: X \rightarrow X^{\prime}$ be a continuous map between pointed spaces, and assume $H^{\bullet}(f)$ is $q$-connected, for some $q \geqslant 1$. Let $A$ be a connected CDGA, and suppose $A$ is a q-model for $X$. Then $\Omega(f) \simeq_{q} \mathrm{id}_{A}$ in $\mathrm{ACDGA}_{0}$.

Proof. Consider the following commuting diagram in $\mathrm{ACDGA}_{0}$,

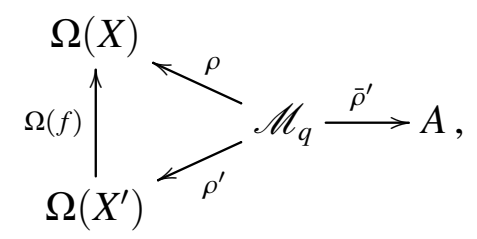

where $\rho^{\prime}$ and $\bar{\rho}^{\prime}$ are $q$-minimal model maps provided by the assumption that $\Omega\left(X^{\prime}\right) \simeq_{q}$ $\Omega(X) \simeq_{q} A$. Clearly, the map $\rho=\Omega(f) \circ \rho^{\prime}$ is a $q$-equivalence, since both $\Omega(f)$ and $\rho^{\prime}$ are. Hence, $\Omega(f) \simeq_{q} \mathrm{id}_{A}$ in $\mathrm{ACDGA}_{0}$, and the claim follows.

Corollary 6.7. Fix $q \geqslant 1$. Let $f: X \rightarrow X^{\prime}$ be a $(q-1)$-connected map between q-finite, pointed spaces, such that $H^{\bullet}(f)$ is q-connected. Let $A$ be a q-finite CDGA, and suppose $A$ is a $q$-model for $X$. Then the conclusions of Corollary 6.5 hold for $A^{\prime}=A$ and $\Phi=\mathrm{id}_{A}$.

We conclude with one more class of spaces and maps where Corollary 6.5 applies. 
Proposition 6.8. Let $f: X \rightarrow X^{\prime}$ be a $(q-1)$-connected map between q-finite, pointed spaces, for some $q \geqslant 1$. Assume that $f$ is formal over $\mathbb{k}$. Then the conclusions of Corollary 6.5 hold for $\Phi=H^{\bullet}(f): H^{\bullet}\left(X^{\prime}, \mathbb{k}\right) \rightarrow H^{\bullet}(X, \mathbb{k})$.

Proof. Our connectivity hypothesis implies that $H^{1}(f)$ is injective. The claim follows at once from Proposition 3.4.

\section{KÄHLER MANIFOLDS}

In this section we show that pointed holomorphic maps between compact Kähler manifolds can be uniformly modeled by the homomorphisms induced in (real) cohomology. As an application, we derive a structural result on the germs at the origin of rank 2 embedded jump loci of Kähler groups.

7.1. Essentially rank one flat connections. We start with some preliminary lemmas.

Lemma 7.1. A non-abelian Lie subalgebra $\mathfrak{g} \subseteq \mathfrak{s l}_{2}(\mathbb{C})$ is either equal to $\mathfrak{s l}_{2}(\mathbb{C})$ or is isomorphic to the standard Borel subalgebra, $\mathfrak{s D l}_{2}(\mathbb{C})$.

Proof. Easy exercise.

We now recall a few facts from [22]. Let $A$ be a CDGA, let $g$ be a Lie algebra, and let $\mathscr{F}(A, \mathfrak{g}) \subset A^{1} \otimes \mathfrak{g}$ be the set of $\mathfrak{g}$-valued flat connections on $A$. Let us define $\mathscr{F}^{1}(A, \mathfrak{g})$ to be the subset of $A^{1} \otimes \mathfrak{g}$ consisting of all tensors of the form $\eta \otimes g$ with $d \eta=0$. We also fix a finite-dimensional representation $\theta: \mathfrak{g} \rightarrow \mathfrak{g l}(V)$, and define $\Pi(A, \theta)$ to be the subset of $\mathscr{F}^{1}(A, \mathfrak{g})$ consisting of all tensors as above which also satisfy $\operatorname{det}(\theta(g))=0$. When $A$ is 1-finite and $\mathfrak{g}$ is finite-dimensional, both $\mathscr{F}^{1}(A, \mathfrak{g})$ and $\Pi(A, \theta)$ are closed, homogeneous subvarieties of $\mathscr{F}(A, \mathfrak{g})$. Moreover, if $H^{i}(A) \neq 0$, then $\Pi(A, \theta) \subseteq \mathscr{R}_{1}^{i}(A, \theta)$.

Lemma 7.2. Under the above finiteness assumptions, every CDGA map $\Phi: A^{\prime} \rightarrow A$ induces algebraic maps, $\Phi \otimes \mathrm{id}: \mathscr{F}^{1}\left(A^{\prime}, \mathfrak{g}\right) \rightarrow \mathscr{F}^{1}(A, \mathfrak{g})$ and $\Phi \otimes \mathrm{id}: \Pi\left(A^{\prime}, \theta\right) \rightarrow \Pi(A, \theta)$. Moreover, if $H^{1}(\Phi)$ is an isomorphism, then both these algebraic maps are isomorphisms.

Proof. Follows directly from the definitions.

Lemma 7.3. Let $\Phi: A^{\prime} \rightarrow A$ be a CDGA map, where $A^{\prime}=\left(\bigwedge^{\bullet} U, d=0\right)$ with $0<$ $\operatorname{dim} U<\infty$ and $A$ is 1 -finite, and assume $H^{1}(\Phi)$ is an isomorphism. Also let $\mathfrak{g} \subseteq \mathfrak{s l}_{2}(\mathbb{C})$ be a Lie subalgebra, and let $\theta: \mathfrak{g} \rightarrow \mathfrak{g l}(V)$ be a finite-dimensional representation. Then the following hold:

(1) $\Phi \otimes \mathrm{id}$ induces an isomorphism between $\mathscr{F}\left(A^{\prime}, \mathfrak{g}\right)$ and $\mathscr{F}^{1}(A, \mathfrak{g})$.

(2) $\Phi \otimes \mathrm{id}$ induces an isomorphism between $\mathscr{R}_{1}^{1}\left(A^{\prime}, \theta\right)$ and $\Pi(A, \theta)$.

Proof. By construction, $A^{\prime}$ is the Chevalley-Eilenberg cochain CDGA of the abelian Lie algebra $U$. Hence, by [22, Lem. 4.14], we have that $\mathscr{F}\left(A^{\prime}, \mathfrak{g}\right)=\mathscr{F}^{1}\left(A^{\prime}, \mathfrak{g}\right)$. The first claim follows at once from Lemma 7.2. 
It is easily checked that $\mathscr{R}_{1}^{1}\left(A^{\prime}\right)=\{0\}$. Since $\mathscr{F}\left(A^{\prime}, \mathfrak{g}\right)=\mathscr{F}^{1}\left(A^{\prime}, \mathfrak{g}\right)$, we may apply [22, Cor. 3.8] to conclude that $\mathscr{R}_{1}^{1}\left(A^{\prime}, \theta\right)=\Pi\left(A^{\prime}, \theta\right)$. The second claim now follows from Lemma 7.2.

7.2. The uniformity property. Let $\left\{f: M \rightarrow M_{f}\right\}_{f \in E}$ be a finite family of pointed, holomorphic maps between compact Kähler manifolds. Each map $f \in E$ induces a homomorphism $H^{\bullet}(f): H^{\bullet}\left(M_{f}\right) \rightarrow H^{\bullet}(M)$ between the respective cohomology algebras with coefficients in $\mathbb{K}=\mathbb{R}$ or $\mathbb{C}$. In fact, these homomorphisms may be viewed as $\mathrm{ACDGA}_{0}$ maps, by setting the differentials to be zero, and taking the augmentations given by the basepoints.

Proposition 7.4. In the above setup, $\Omega(f) \simeq H^{\bullet}(f)$ in $\mathrm{ACDGA}_{0}$, uniformly with respect to $f \in E$.

Proof. To prove the claim, it is enough to show there is a functorial zig-zag of quasiisomorphisms in ACDGA connecting $\Omega(M)$ to $H^{\bullet}(M)$, for any pointed, compact Kähler manifold $M$. In order to construct such a zig-zag, we proceed in two steps, following [7, $\S 6]$ and $[13, \S 11]$. It is enough to work over $\mathbb{K}=\mathbb{R}$.

Let $\left(\Omega_{\mathrm{dR}}(M), d\right)$ be the de Rham CDGA of the underlying differentiable manifold, with $d$ the exterior differential. Set $d^{c}=J^{-1} d J$, where $J$ is the complex structure on the tangent bundle to $M$. This gives another CDGA, $\left(\Omega_{\mathrm{dR}}(M), d^{c}\right)$. By the first proof of the Main Theorem from [7], there is a zig-zag of quasi-isomorphisms in CDGA connecting $\left(\Omega_{\mathrm{dR}}(M), d\right)$ to $\left(H^{\bullet}\left(\Omega_{\mathrm{dR}}(M), d^{c}\right), d=0\right)$, natural with respect to holomorphic maps. Taking homomorphisms induced in cohomology, we obtain a natural zig-zag of CDGA quasi-isomorphisms connecting $\left(H_{\mathrm{dR}}^{\bullet}(M), d=0\right)$ to $\left(H^{\bullet}\left(\Omega_{\mathrm{dR}}(M), d^{c}\right), d=0\right)$. In fact, both zig-zags are in ACDGA, since all their terms are equal to $\mathbb{R}$ when $M$ is a point. Combining these two zig-zags, we obtain a functorial zig-zag of quasi-isomorphisms in ACDGA from $\Omega_{\mathrm{dR}}(M)$ to $\left(H_{\mathrm{dR}}^{\bullet}(M), d=0\right)$.

On the other hand, the proof of the de Rham theorem from [13] provides a zig-zag of quasi-isomorphisms in CDGA connecting $\Omega_{\mathrm{dR}}(M)$ to $\Omega_{\mathbb{R}}(M)$, which is natural with respect to differentiable maps. An argument as above shows that this zig-zag is in ACDGA. Putting things together, and using the classical de Rham theorem, we arrive at the desired conclusion.

7.3. Admissible maps and rank 1 jump loci. A connected, complex manifold $M$ is said to be a quasi-compact Kähler manifold if there is compact Kähler manifold $\bar{M}$ and a normal crossing divisor $D \subset M$ such that $M=\bar{M} \backslash D$. Of course, all compact Kähler manifolds belong to this class. Furthermore, if $M$ is an irreducible smooth, complex quasiprojective variety, or, for short, a quasi-projective manifold, then $M$ is also of this type, by resolution of singularities.

Given a quasi-compact Kähler manifold $M$, there is a certain finite family of pointed holomorphic maps, $\left\{f: M \rightarrow M_{f}\right\}_{f \in \mathscr{E}(M)}$, with each $M_{f}$ a quasi-projective manifold, which is intimately related to the structure near 1 of the characteristic variety $\mathscr{V}_{1}^{1}(M)$. 
More precisely, a holomorphic map onto a smooth complex curve, $f: M \rightarrow M_{f}$, is said to be admissible if it extends to a holomorphic surjection with connected fibers, $\bar{f}: \bar{M} \rightarrow \bar{M}_{f}$, where $\bar{M}$ (respectively $\bar{M}_{f}$ ) is a Kähler compactification of $M$ (respectively $M_{f}$ ) obtained by adding a normal crossing divisor. It is known that, up to reparametrization at the target, there is a finite family $\mathscr{E}(M)$ of such maps with the property that $\chi\left(M_{f}\right)<0$. For each $f \in \mathscr{E}(M)$, let us write $\pi=\pi_{1}(M)$ and $\pi_{f}=\pi_{1}\left(M_{f}\right)$. It is readily seen that the induced homomorphism on fundamental groups, $f_{\sharp}: \pi \rightarrow \pi_{f}$, is surjective. Work of Arapura [2] shows that the correspondence

$$
f \sim f_{\sharp}^{!} \operatorname{Hom}\left(\pi_{f}, \mathbb{C}^{\times}\right)
$$

establishes a bijection between the set $\mathscr{E}(M)$ and the set of positive-dimensional, irreducible components of the characteristic variety $\mathscr{V}_{1}^{1}(M)$ passing through 1.

For a pointed CW-space $M$ with fundamental group $\pi$, we denote by $f_{0}: M \rightarrow K\left(\pi_{\mathrm{abf}}, 1\right)$ the classifying map determined up to homotopy by the property that $\left(f_{0}\right)_{\sharp}=$ abf: $\pi \rightarrow$ $\pi_{\mathrm{abf}}$, where abf is the canonical projection of the group $\pi$ onto its maximal torsion-free abelian quotient. When $M$ is a quasi-compact Kähler manifold, we set

$$
E(M)=\mathscr{E}(M) \cup\left\{f_{0}\right\} .
$$

In the rank one case, i.e., when $\iota=\mathrm{id}_{\mathbb{C}^{\times}}$, both inclusions, (5.14) and (5.15) for $i=r=$ 1 , become equalities near the origin 1 , for the family $\left\{f_{\sharp} \mid f \in E(M)\right\}$.

Theorem 7.5. Let $M$ be a quasi-compact Kähler manifold, and let $\pi=\pi_{1}(M)$. Then,

$$
\begin{aligned}
\operatorname{Hom}\left(\pi, \mathbb{C}^{\times}\right)_{(1)} & =\bigcup_{f \in E(M)} f_{\sharp}^{!} \operatorname{Hom}\left(\pi_{f}, \mathbb{C}^{\times}\right)_{(1)}, \\
\mathscr{V}_{1}^{1}(\pi)_{(1)} & =\bigcup_{f \in E(M)} f_{\sharp}^{!} \mathscr{V}_{1}^{1}\left(\pi_{f}\right)_{(1)} .
\end{aligned}
$$

Proof. The first claim is easily verified. Indeed, the abelianization map, $\pi \rightarrow \pi_{\mathrm{ab}}$, induces an isomorphism of character groups, while the map induced by the natural projection $\pi_{\mathrm{ab}} \rightarrow \pi_{\mathrm{abf}}$ identifies $\operatorname{Hom}\left(\pi_{\mathrm{abf}}, \mathbb{C}^{\times}\right)$with the identity component of $\operatorname{Hom}\left(\pi_{\mathrm{ab}}, \mathbb{C}^{\times}\right)$. It follows that $\left(f_{0}\right)_{\sharp}=\pi_{\mathrm{abf}}$ induces an isomorphism between germs at 1 of $\mathbb{C}^{\times}$-representation varieties, and so (7.3) holds.

The second claim is much more subtle. Since $\chi\left(M_{f}\right)<0$, it is easily seen that $\mathscr{V}_{1}^{1}\left(\pi_{f}\right)=\mathscr{V}_{1}^{1}\left(M_{f}\right)=\operatorname{Hom}\left(\pi_{f}, \mathbb{C}^{\times}\right)$, for $f \in \mathscr{E}(M)$. If $b_{1}(M)=0$, we know from (5.5) that $\mathscr{V}_{1}^{1}(\pi)_{(1)}=\varnothing$, and we are done. If $b_{1}(M)>0$, then either $\mathscr{V}_{1}^{1}(\pi)_{(1)}=\{1\}$, or all irreducible components of $\mathscr{V}_{1}^{1}(\pi)$ passing through 1 are positive-dimensional. In the first case we are done, since $1 \in\left(f_{0}\right)_{\sharp} \mathscr{V}_{1}^{1}\left(\pi_{\mathrm{abf}}\right)$. In the second case, equality (7.4) follows from the aforementioned deep results of Arapura. This completes the proof.

7.4. Rank 2 embedded jump loci of Kähler manifolds. Let $M$ be a compact Kähler manifold with fundamental group $\pi$. A map $f: M \rightarrow M_{f}$ is admissible in the sense from $§ 7.3$ if $M_{f}$ is a compact Riemann surface and $f$ is a holomorphic surjection with 
connected fibers. The Albanese map, $f_{0}: M \rightarrow \mathrm{Alb}(M)$, is a holomorphic map between compact Kähler manifolds which classifies the canonical projection, $\pi \rightarrow \pi_{\text {abf }}$.

Our next goal is to extend the rank 1 results (7.3)-(7.4) to the rank 2 case. We start with a lemma.

Lemma 7.6. Let $M$ be a compact Kähler manifold with $b_{1}(M)>0$. Let $\mathfrak{g}$ be a non-abelian Lie subalgebra of $\mathfrak{s l}_{2}(\mathbb{C})$, and let $\theta: \mathfrak{g} \rightarrow \mathfrak{g l}(V)$ be a finite-dimensional representation. Then the following equalities hold:

$$
\begin{gathered}
\mathscr{F}\left(H^{\bullet}(M), \mathfrak{g}\right)=\mathscr{F}^{1}\left(H^{\bullet}(M), \mathfrak{g}\right) \cup \bigcup_{f \in \mathscr{E}(M)} f^{!}\left(\mathscr{F}\left(H^{\bullet}\left(M_{f}\right), \mathfrak{g}\right)\right), \\
\mathscr{R}_{1}^{1}\left(H^{\bullet}(M), \theta\right)=\Pi\left(H^{\bullet}(M), \theta\right) \cup \bigcup_{f \in \mathscr{E}(M)} f^{!}\left(\mathscr{F}\left(H^{\bullet}\left(M_{f}\right), \mathfrak{g}\right)\right),
\end{gathered}
$$

where all CDGAs are endowed with zero differential.

Proof. This is proved in [22, Cor. 7.2] for a 1-formal, quasi-projective manifold $M$. By results from [10], the proof also works for 1-formal, quasi-compact Kähler manifolds, in particular, for a compact Kähler manifold $M$.

Theorem 7.7. Let $M$ be a compact Kähler manifold with fundamental group $\pi$, and set $E(M)=\mathscr{E}(M) \cup\left\{f_{0}\right\}$ as in (7.2). Let $G$ be $\mathbb{C}$-linear algebraic group with non-abelian Lie algebra $\mathfrak{g} \subseteq \mathfrak{s l}_{2}(\mathbb{C})$, and let $\iota: G \rightarrow \mathrm{GL}(V)$ be a rational representation. Then,

$$
\operatorname{Hom}(\pi, G)_{(1)}=\bigcup_{f \in E(M)} f_{\sharp}^{!} \operatorname{Hom}\left(\pi_{f}, G\right)_{(1)},
$$

and, for $i=r=1$ or $i=0$ and $r \geqslant 1$,

$$
\mathscr{V}_{r}^{i}(\pi, \iota)_{(1)}=\bigcup_{f \in E(M)} f_{\sharp}^{!} \mathscr{V}_{r}^{i}\left(\pi_{f}, \iota\right)_{(1)} .
$$

Proof. We wish to apply Theorem 6.4 with $q=1$ to the family of pointed maps $\{f: M \rightarrow$ $\left.M_{f}\right\}_{f \in E(M)}$ and CDGA maps $\Phi_{f}=\left\{H^{\bullet}(f): H^{\bullet}\left(M_{f}\right) \rightarrow H^{\bullet}(M)\right\}_{f \in E(M)}$, where all the differentials are set equal to 0 . For that, we need to verify that the three hypotheses of the theorem hold.

First, all spaces and CDGAs in question are 1-finite (in fact, $\infty$-finite). Second, each map $f_{\sharp}$ is surjective, hence each $f \in E(M)$ is 0-connected. Thus, $H^{\bullet}(f)$ is also 0-connected. Finally, by Proposition 7.4, $\Omega(f) \simeq H^{\bullet}(f)$ in $\mathrm{ACDGA}_{0}$, uniformly with respect to $f \in$ $E(M)$.

In the case when $i=0$, equality (7.7) clearly implies equality (7.8), by Corollary 5.8. Thus, we may assume $i=r=1$ in (7.8).

Suppose now that $b_{1}(M)=0$. By (5.5), we have that $\mathscr{V}_{1}^{1}(\pi, \iota)_{(1)}=\varnothing$. Therefore, equality (7.8) follows trivially. Moreover, the natural map $\Omega(K(1,1)) \rightarrow \Omega(K(\pi, 1))$ is a 
1-equivalence; hence, $\pi$ has the same 1-minimal model as the trivial group. It then follows from [9, Thm. A] that $\operatorname{Hom}(\pi, G)_{(1)}=\{1\}$. Therefore, equality (7.7) holds trivially.

Thus, we may also assume that $b_{1}(M)>0$. We deduce from formula (7.5) and Lemma 7.3, part (1) that

$$
\mathscr{F}\left(H^{\bullet}(M), \mathfrak{g}\right)=f_{0}^{!} \mathscr{F}\left(H^{\bullet}\left(M_{0}\right), \mathfrak{g}\right) \cup \bigcup_{f \in \mathscr{E}(M)} f^{!}\left(\mathscr{F}\left(H^{\bullet}\left(M_{f}\right), \mathfrak{g}\right)\right),
$$

where $M_{0}$ denotes the Albanese variety $\operatorname{Alb}(M) \simeq K\left(\pi_{\mathrm{abf}}, 1\right)$, and $H^{\bullet}\left(M_{0}\right)=\bigwedge^{\bullet} H^{1}(M)$. By taking germs at the origin and using the naturality properties from Theorem 6.4 , formula (7.9) implies that equality (7.7) holds.

Similarly, formula (7.6) and Lemma 7.3 part (2) together imply that

$$
\mathscr{R}_{1}^{1}\left(H^{\bullet}(M), \theta\right)=f_{0}^{!} \mathscr{R}_{1}^{1}\left(H^{\bullet}\left(M_{0}\right), \theta\right) \cup \bigcup_{f \in \mathscr{E}(M)} f^{!}\left(\mathscr{F}\left(H^{\bullet}\left(M_{f}\right), \mathfrak{g}\right)\right) .
$$

For each $f \in \mathscr{E}(M)$, note that $M_{f}$ is a 2-dimensional CW-complex with $\chi\left(M_{f}\right)<0$. An easy Euler characteristic argument then shows that $\mathscr{V}_{1}^{1}\left(\pi_{f}, \iota\right)=\mathscr{V}_{1}^{1}\left(M_{f}, \iota\right)=\operatorname{Hom}\left(\pi_{f}, G\right)$. Again by Theorem 6.4, formula (7.10) now implies that equality (7.8) holds. This completes the proof.

Remark 7.8. In [20, Cor. B], Loray, Pereira, and Touzet prove the following result, which refines earlier results of Corlette and Simpson [6]. Let $X$ be a quasi-projective manifold, and let $\rho \in \operatorname{Hom}\left(\pi_{1}(X), \mathrm{SL}_{2}(\mathbb{C})\right)$ be a representation which is not virtually abelian. Then there is an orbifold morphism, $f: X \rightarrow Y$, such that the associated representation, $\tilde{\rho} \in \operatorname{Hom}\left(\pi_{1}(X), \mathrm{PSL}_{2}(\mathbb{C})\right)$, belongs to $f_{\sharp}^{!} \operatorname{Hom}\left(\pi_{1}^{\text {orb }}(Y), \operatorname{PSL}_{2}(\mathbb{C})\right)$, where $Y$ is either a 1-dimensional complex orbifold, or a polydisk Shimura modular orbifold.

For a finitely generated group $\pi$ and a linear algebraic group $G$, the abelian part of the representation variety $\operatorname{Hom}(\pi, G)$ coincides near 1 with $\operatorname{abf}^{!} \operatorname{Hom}\left(\pi_{\mathrm{abf}}, G\right)_{(1)}$. Indeed, [9, Thm. A] implies that the canonical projection $\pi_{\mathrm{ab}} \rightarrow \pi_{\mathrm{abf}}$ induces an isomorphism of germs at the origin of the respective representation varieties.

This remark shows that formula (7.7) from Theorem 7.7 may be viewed as a compact Kähler analogue near 1 of [20, Cor. B]. In this context, it provides a simpler classification: the representation $\rho$ is either abelian, or it pulls back via an admissible map from a compact Riemann surface of genus $g>1$.

7.5. The main difficulty in the non-abelian case. The naturality property from $[9$, Thm. $\mathrm{B}(2)]$ is a consequence of the following fact, which holds in the abelian case. Let $X$ be a 1-finite space and $A^{\bullet}$ a 1 -finite CDGA. The fact that $\Omega_{\mathbb{k}}(X) \simeq_{1} A$ in CDGA means that there is a zig-zag of 1-equivalences in CDGA,

$$
\Omega_{\mathbb{k}}(X) \stackrel{\psi}{\longleftarrow} \mathcal{N} \stackrel{\bar{\psi}}{\longrightarrow} A,
$$

where $\mathcal{N}=\left(\wedge^{\bullet} U, d\right)$ is a 1-minimal CDGA, see Lemma 3.3. If $\mathfrak{g}$ is an abelian Lie algebra, it follows from the definitions that $\mathscr{F}(B, \mathfrak{g})=H^{1}(B) \otimes \mathfrak{g}$, for any connected CDGA $B$. 
Applying this observation to the map $\bar{\psi}$, we conclude that, in the abelian case, there is a bijection

$$
\bar{\psi} \otimes \mathrm{id}: \mathscr{F}(\mathcal{N}, \mathfrak{g}) \stackrel{\simeq}{\longrightarrow} \mathscr{F}(A, \mathfrak{g}) .
$$

For a non-abelian Lie algebra $\mathfrak{g}$, though, this map is not necessarily surjective. To illustrate this phenomenon, we first need a lemma.

Lemma 7.9. If $\mathfrak{g}=\mathfrak{s l}_{2}(\mathbb{C})$ and $\bar{\psi} \otimes \mathrm{id}$ is surjective, then $\mathscr{F}(A, \mathfrak{g})=\mathscr{F}^{1}(A, \mathfrak{g})$.

Proof. The CDGA $\mathcal{N}=\left(\bigwedge^{\bullet} U, d\right)$ comes endowed with the canonical filtration, $\mathcal{N}=$ $\bigcup_{n \geqslant 1} \mathcal{N}_{n}$, of a 1-minimal CDGA, where each CDGA $\mathcal{N}_{n}$ is of the form $\left(\wedge^{\bullet} U_{n}, d\right)$. Since $\operatorname{dim} H^{1}(\mathcal{N})<\infty, \mathscr{N}_{n}$ is the cochain algebra of a certain finite-dimensional, nilpotent Lie algebra. Since $\mathfrak{g}=\mathfrak{s l}_{2}(\mathbb{C})$, it follows from [22, Lem. 4.14] that $\mathscr{F}\left(\mathcal{N}_{n}, \mathfrak{g}\right)=\mathscr{F}^{1}\left(\mathcal{N}_{n}, \mathfrak{g}\right)$, for each $n \geqslant 1$. Hence, $\mathscr{F}(\mathcal{N}, \mathfrak{g})=\mathscr{F}^{1}(\mathcal{N}, \mathfrak{g})$. Since $\bar{\psi} \otimes$ id is surjective, we conclude that $\mathscr{F}(A, \mathfrak{g})=\mathscr{F}^{1}(A, \mathfrak{g})$.

Example 7.10. Let $\Sigma_{g}$ be a compact Riemann surface of genus $g>1$. Since $\Sigma_{g}$ is a formal space, the CDGA $A=\left(H^{\bullet}\left(\Sigma_{g}\right), d=0\right)$ is a finite model for it. Let $\mathcal{N}$ be a 1-minimal model for $A$, and let $\bar{\psi}: \mathcal{N} \rightarrow A$ be the corresponding map. It follows from [22, Lem. 7.3] that $\mathscr{F}(A, \mathfrak{g}) \neq \mathscr{F}^{1}(A, \mathfrak{g})$. By Lemma 7.9 , then, the map $\bar{\psi} \otimes$ id is not surjective.

Thus, in the case when $G=\mathrm{SL}_{2}(\mathbb{C})$, we have no natural analytic map $\mathscr{F}(A, \mathfrak{g}) \rightarrow$ $\operatorname{Hom}\left(\pi_{1}(X), G\right)$. This is the reason why we have to construct a local analytic isomorphism between the germs at the origin of the two varieties, in a manner which is compatible with both continuous maps and CDGA maps, using the simultaneous Artin approximation technique from Proposition 2.3.

\section{PRincipal BUndLes}

In this section, we apply our theory to principal bundles. When the base manifold is formal, we obtain a structural result for the germs at the origin of rank 2 embedded jump loci of the total space.

8.1. Two-element families with the uniform property. In the applications of Theorem 6.4 , we also need to take into account the projection of a group $\pi$ onto its maximal torsionfree abelian quotient, abf: $\pi \rightarrow \pi_{\mathrm{abf}}$.

Theorem 8.1. Let $f: M \rightarrow N$ be a continuous, pointed map. Denote by $f_{0}: M \rightarrow$ $K\left(\pi_{1}(M)_{\mathrm{abf}}, 1\right)$ the classifying map for the above projection. Suppose that $M$ and $N$ are $q$-finite, for some $q \geqslant 1$, and that $\Omega(f) \simeq_{q} \Phi$ in $\mathrm{ACDGA}_{0}$, where $\Phi: A_{N} \rightarrow A_{M}$ is a CDGA map between q-finite objects. Set $A_{0}^{\bullet}=\left(\bigwedge^{\bullet} H^{1}(M), d=0\right)$. There is then a CDGA map $\Phi_{0}: A_{0} \rightarrow A_{M}$ inducing an isomorphism on $H^{1}$, and such that $\Omega\left(f_{0}\right) \simeq_{q} \Phi_{0}$ in $\mathrm{ACDGA}_{0}$, uniformly with respect to the families $\left\{f, f_{0}\right\}$ and $\left\{\Phi, \Phi_{0}\right\}$. Moreover, if $f$ and $\Phi$ are 0 connected maps, then all the hypotheses from Theorem 6.4 are satisfied for $q=1$. 
Proof. The assumption that $\Omega(f) \simeq_{q} \Phi$ provides a zig-zag $Z$ of $q$-equivalences in ACDGA connecting $\Omega_{\mathbb{K}}(M)$ to $A_{M}$. Let $\rho: \mathcal{N} \rightarrow \Omega_{\mathbb{k}}(M)$ be a $\pi_{1}$-adapted 1-minimal model map, as in $[9, \S 6.4]$. This map can be extended to a $q$-minimal model map $\rho: \mathscr{M} \rightarrow \Omega_{\mathbb{k}}(M)$. By Proposition 4.6, there is a special zig-zag $S$ of the form $\Omega_{\mathbb{k}}(M) \stackrel{\rho}{\longleftarrow} \mathscr{M} \stackrel{\bar{\rho}}{\longrightarrow} A_{M}$ such that $\beta_{Z}=\beta_{S}$.

Now, as explained in $[9, \S 6.4]$, there is a canonical CDGA inclusion, $j: A_{0} \hookrightarrow \mathcal{N}$, inducing an isomorphism on $H^{1}$. It follows that the map $\Phi_{0}=\bar{\rho} \circ j: A_{0} \rightarrow A_{M}$ has the same property. Putting things together, we obtain the following commuting diagram in ACDGA:

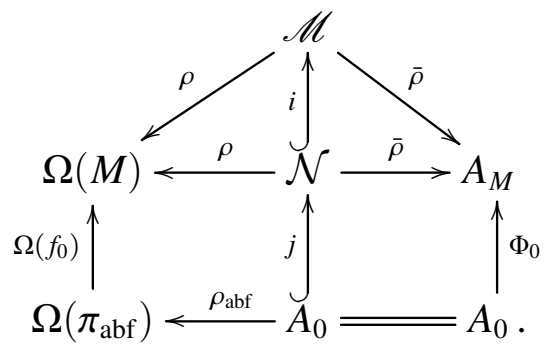

Both upper-diagonal arrows are $q$-equivalences, and both lower-horizontal arrows are $\infty$-equivalences. It follows that $\Omega\left(f_{0}\right) \simeq_{q} \Phi_{0}$ in $\mathrm{ACDGA}_{0}$, as claimed. The uniform property follows from the equality $\beta_{Z}=\beta_{S}$. It is obvious that the map $f_{0}$ is 0 -connected. Finally, since $H^{1}\left(\Phi_{0}\right)$ is injective, the map $\Phi_{0}$ is also 0 -connected.

8.2. Models for principal bundle projections. Let $K$ be a compact, connected real Lie group acting freely on a closed, smooth manifold $M$. Let $N=M / K$ be the orbit space, and let $f: M \rightarrow N$ be the projection map of the resulting principal $K$-bundle. Of course, both $M$ and $N$ have the homotopy type of a finite $\mathrm{CW}$-complex. We will fix compatible basepoints for $M$ and $N$. Note that $f$ is 0 -connected, by the exact homotopy sequence of the fibration $K \rightarrow M \stackrel{f}{\rightarrow} N$ and the connectivity of $K$.

By a classical result of $\mathrm{H}$. Hopf, the cohomology algebra of $K$ (with coefficients in a field $\mathbb{k}$ of characteristic 0 ) is of the form $H^{\bullet}(K)=\bigwedge P^{\bullet}$, where $P^{\bullet}$ is a finite-dimensional, oddly graded $\mathbb{k}$-vector space. Let $[\tau]: P^{\bullet} \rightarrow H^{\bullet+1}(N)$ be the transgression in the Serre spectral sequence of our fibration.

Suppose $A_{N}$ is a CDGA model for $N$, so that there is a zig-zag of quasi-isomorphisms connecting $\Omega_{\mathbb{k}}(N)$ to $A_{N}$. Such a zig-zag yields an isomorphism of $H^{\bullet}(N)$ with $H^{\bullet}\left(A_{N}\right)$. Let $\tau: P^{\bullet} \rightarrow Z^{\bullet+1}\left(A_{N}\right)$ be a lift of $[\tau]$. As noted in $\S 3.3$, the Hirsch extension $A_{M}:=$ $A_{N} \otimes_{\tau} \bigwedge P$ is well-defined, up to a CDGA isomorphism extending $\mathrm{id}_{A_{N}}$.

Proposition 8.2. Let $f: M \rightarrow N$ be the projection map of a principal $K$-bundle as above, and suppose $N$ admits a finite model $A_{N}$. Let $\Phi: A_{N} \hookrightarrow A_{M}$ be the canonical CDGA inclusion. Then $A_{M}$ is a finite model for $M$, and both $f$ and $\Phi$ are 0-connected maps. Moreover, $\Omega(f) \simeq \Phi$ in $\mathrm{ACDGA}_{0}$, and thus the conclusions of Corollary 6.5 hold for $q=1$. 
Proof. Clearly, since $A_{N}$ is a finite CDGA, then $A_{M}$ is also a finite CDGA. Equally clearly, the map $\Phi$ is 0-connected. By the classical Hirsch Lemma (see [13, pp. 216-218]) there is a commutative diagram in ACDGA,

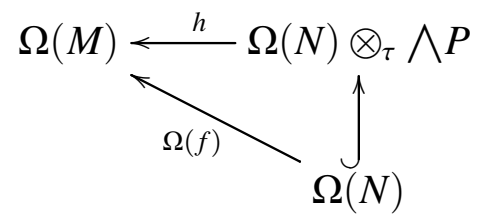

where $h$ is an $\infty$-equivalence. Since by assumption $\Omega(N) \simeq A_{N}$, there is a minimal CDGA $\mathcal{N}$, connected by quasi-isomorphisms $\psi: \mathcal{N} \rightarrow \Omega(N)$ and $\bar{\psi}: \mathcal{N} \rightarrow A_{N}$. We then obtain a commuting diagram in CDGA,

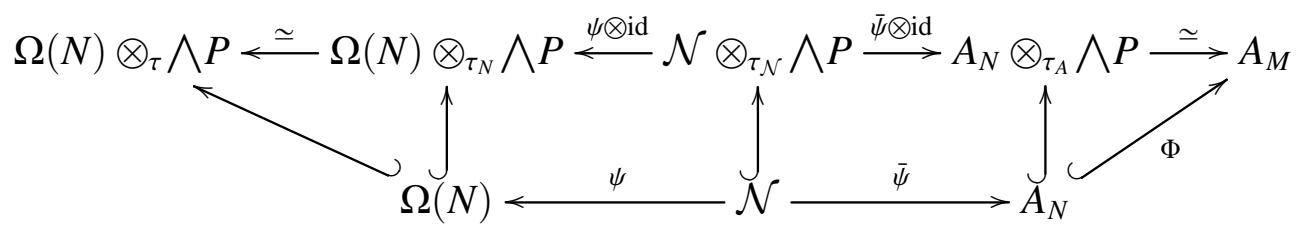

where the transgression $\left[\tau_{\mathcal{N}}\right]$ is identified with $\left[\tau_{N}\right]$ using $H^{\bullet}(\psi)$, while $\left[\tau_{\mathcal{N}}\right]$ is identified with $\left[\tau_{A}\right]$ using $H^{\bullet}(\bar{\psi})$. Note that all CDGAs in (8.3) are augmented, and all maps respect augmentations. By [13, Lem. 14.2], the maps $\psi \otimes$ id and $\bar{\psi} \otimes$ id are quasi-isomorphisms, since both $\psi$ and $\bar{\psi}$ are. Splicing together diagrams (8.2) and (8.3) we reach the desired conclusions.

8.3. Embedded jump loci of principal bundles. Before stating and proving the main result of this section, we need two more lemmas. According to the guiding philosophy of [22], the essentially rank 1 part of the higher-rank resonance varieties of a CDGA is determined by rank 1 resonance. We begin with a version of this general principle, valid for families of CDGA morphisms.

Fix an integer $q \geqslant 1$. Let $\left\{\phi_{f}: A_{f} \rightarrow A\right\}_{f \in \mathscr{E}}$ be a finite family of $(q-1)$-connected maps between connected $\mathbb{C}$-CDGAs. Also, let $\mathfrak{g}$ be a Lie algebra, and let $\theta: \mathfrak{g} \rightarrow \mathfrak{g l}(V)$ be a finite-dimensional representation. For each $i \leqslant q$ such that $H^{i}(A) \neq 0$, Corollary 3.8 and Lemma 2.6 from [22] give an inclusion

$$
\mathscr{R}_{1}^{i}(A, \theta) \supseteq \Pi(A, \theta) \cup \bigcup_{f \in \mathscr{E}}\left(\phi_{f} \otimes \mathrm{id}\right) \mathscr{R}_{1}^{i}\left(A_{f}, \theta\right) .
$$

Lemma 8.3. Assume (8.4) holds as an equality in the rank 1 case. Then

$$
\mathscr{R}_{1}^{i}(A, \theta) \cap \mathscr{F}^{1}(A, \mathfrak{g}) \subseteq \Pi(A, \theta) \cup \bigcup_{f \in \mathscr{E}}\left(\phi_{f} \otimes \mathrm{id}\right) \mathscr{R}_{1}^{i}\left(A_{f}, \theta\right) .
$$

Proof. Let $\omega=\eta \otimes g$ be a non-zero element in $\left(\mathscr{R}_{1}^{i}(A, \theta) \cap \mathscr{F}^{1}(A, \mathfrak{g})\right) \backslash \Pi(A, \theta)$. From [22, Cor. 3.8], we know that $\eta \otimes g$ belongs to $\mathscr{R}_{1}^{i}(A, \theta) \cap \mathscr{F}^{1}(A, \mathfrak{g})$ if and only if there is an eigenvalue $\lambda$ of $\theta(g)$ such that $\lambda \eta \in \mathscr{R}_{1}^{i}(A)$. By our assumption on the rank 1 resonance, 
$\lambda \eta=\phi_{f}\left(\eta^{\prime}\right)$, for some $\eta^{\prime} \in \mathscr{R}_{1}^{i}\left(A_{f}\right)$. Since $\lambda \neq 0$ we infer that $\omega=\left(\phi_{f} \otimes \mathrm{id}\right)\left(\eta_{f} \otimes g\right)$, for some $\eta_{f} \in A_{f}^{1}$ such that $d \eta_{f}=0$ and $\lambda \eta_{f} \in \mathscr{R}_{1}^{i}\left(A_{f}\right)$. Again by [22, Cor. 3.8], we conclude that $\eta_{f} \otimes g$ belongs to $\mathscr{R}_{1}^{i}\left(A_{f}, \theta\right) \cap \mathscr{F}^{1}\left(A_{f}, \mathfrak{g}\right)$, and we are done.

Lemma 8.4. Let $A$ be a 1-finite $\mathbb{C}$-CDGA with $d=0$, and let $\theta: \mathfrak{g} \rightarrow \mathfrak{g l}(V)$ be a finitedimensional representation of a non-abelian Lie subalgebra of $\mathfrak{s l}_{2}(\mathbb{C})$. Then $\mathscr{F}(A, \mathfrak{g})=$ $\mathscr{F}^{1}(A, \mathfrak{g}) \cup \mathscr{R}_{1}^{1}(A, \theta)$.

Proof. Let $\omega \in \mathscr{F}(A, \mathfrak{g}) \backslash \mathscr{F}^{1}(A, \mathfrak{g})$. It follows from the proof of [22, Prop. 5.3] that $\omega \in$ $U \otimes \mathfrak{g}$, where $U \subseteq A^{1}$ is a linear subspace of dimension at least 2 which is isotropic with respect to the multiplication map $A^{1} \wedge A^{1} \rightarrow A^{2}$. Clearly, $A_{U}:=\mathbb{C} \cdot 1 \oplus U$ is a finite subCDGA of $A$, and $\chi\left(H^{\bullet}\left(A_{U}\right)\right)<0$. By [22, Prop. 2.4], we have that $\mathscr{F}\left(A_{U}, \mathfrak{g}\right)=\mathscr{R}_{1}^{1}\left(A_{U}, \theta\right)$. Therefore, $\omega \in \mathscr{R}_{1}^{1}\left(A_{U}, \theta\right) \subseteq \mathscr{R}_{1}^{1}(A, \theta)$, and this completes the proof.

Theorem 8.5. Let $f: M \rightarrow N$ be the projection map of a principal $K$-bundle, where both $M$ and $N$ are smooth, closed manifolds, and $K$ is a compact, connected real Lie group. Let $G$ be a complex linear algebraic group, with non-abelian Lie algebra $\mathfrak{g} \subseteq \mathfrak{s l}_{2}(\mathbb{C})$. Let $\iota: G \rightarrow \mathrm{GL}(V)$ be a rational representation. Let $f_{\sharp}: \pi_{1}(M) \rightarrow \pi_{1}(N)$ be the induced homomorphism on fundamental groups, and let abf: $\pi_{1}(M) \rightarrow \pi_{1}(M)_{\text {abf }}$ be the canonical projection. Suppose $N$ is formal. Then,

$$
\operatorname{Hom}\left(\pi_{1}(M), G\right)_{(1)}=\operatorname{abf}^{!} \operatorname{Hom}\left(\pi_{1}(M)_{\mathrm{abf}}, G\right)_{(1)} \cup f_{\sharp}^{!} \operatorname{Hom}\left(\pi_{1}(N), G\right)_{(1)},
$$

and, for $i=r=1$ or $i=0$ and $r \geqslant 1$,

$$
\mathscr{V}_{r}^{i}\left(\pi_{1}(M), \iota\right)_{(1)}=\operatorname{abf}^{!} \mathscr{V}_{r}^{i}\left(\pi_{1}(M)_{\mathrm{abf}}, \iota\right)_{(1)} \cup f_{\sharp}^{!} \mathscr{V}_{r}^{i}\left(\pi_{1}(N), \iota\right)_{(1)} .
$$

Proof. By Corollary 5.8, equality of germs at 1 in (5.14) implies equality at 1 in (5.15) for $i=0$ and $r \geqslant 1$. Thus, in order to verify equality (8.6), it is enough to assume $i=r=1$. As we saw in the proof of Theorem 7.7, both our claims hold trivially when $b_{1}(M)=0$. Consequently, we may also assume that $b_{1}(M)>0$.

Since the orbit space $N$ is formal, we may take as a model for it the CDGA $A_{N}=$ $\left(H^{\bullet}(N), d=0\right)$. As usual, let $[\tau]: P^{\bullet} \rightarrow H^{\bullet+1}(N)$ be the transgression in the Serre spectral sequence of the fibration $K \rightarrow M \rightarrow N$. By Proposition 8.2, the Hirsch extension $A_{M}:=A_{N} \otimes_{\tau} \bigwedge P$ is a finite CDGA model for $M$, and the canonical inclusion $\Phi: H^{\bullet}(N) \hookrightarrow$ $A_{M}^{\bullet}$ is a model for the map $\Omega(f): \Omega(N) \rightarrow \Omega(M)$.

Now set $A_{0}^{\bullet}=\left(\bigwedge^{\bullet} H^{1}(M), d=0\right)$, and let $f_{0}: M \rightarrow K\left(\pi_{1}(M)_{\text {abf }}, 1\right)$ be the canonical map defined by the homomorphism abf. By Theorem 8.1 (with $q=1$ ), there is a CDGA map $\Phi_{0}: A_{0} \rightarrow A_{M}$ such that $\Omega\left(f_{0}\right) \simeq_{1} \Phi_{0}$ and $\Omega(f) \simeq_{1} \Phi$ in ACDGA $_{0}$, uniformly with respect to the families $\left\{f, f_{0}\right\}$ and $\left\{\Phi, \Phi_{0}\right\}$. Since, as was mentioned in Proposition 8.2, both $f$ and $\Phi$ are 0 -connected, Theorem 6.4 applies, giving an analytic isomorphism of 
embedded germs,

$$
\begin{aligned}
\left(\operatorname{Hom}\left(\pi_{1}(M), G\right), \text { abf }^{!} \operatorname{Hom}\left(\pi_{1}(M)_{\mathrm{abf}}, G\right) \cup f_{\sharp}^{!} \operatorname{Hom}\left(\pi_{1}(N), G\right)\right)_{(1)} \cong \\
\left(\mathscr{F}\left(A_{M}, \mathfrak{g}\right),\left(\Phi_{0} \otimes \mathrm{id}\right) \mathscr{F}\left(A_{0}, \mathfrak{g}\right) \cup(\Phi \otimes \mathrm{id}) \mathscr{F}\left(A_{N}, \mathfrak{g}\right)\right)_{(0)} .
\end{aligned}
$$

On the other hand, Proposition 5.3 from [28] guarantees the global equality

$$
\mathscr{F}\left(A_{M}, \mathfrak{g}\right)=\mathscr{F}^{1}\left(A_{M}, \mathfrak{g}\right) \cup(\Phi \otimes \mathrm{id}) \mathscr{F}\left(A_{N}, \mathfrak{g}\right) .
$$

We may also apply Lemma 7.3 to the map $\Phi_{0}$ to deduce the global equalities

$$
\begin{aligned}
\mathscr{F}^{1}\left(A_{M}, \mathfrak{g}\right) & =\left(\Phi_{0} \otimes \mathrm{id}\right) \mathscr{F}\left(A_{0}, \mathfrak{g}\right), \\
\Pi\left(A_{M}, \theta\right) & =\left(\Phi_{0} \otimes \mathrm{id}\right) \mathscr{R}_{1}^{1}\left(A_{0}, \theta\right) .
\end{aligned}
$$

Using equations (8.7)-(8.9) as well as Theorem 6.4, we see that, in order to complete the proof, it is enough to show the following: if the inclusion

$$
\mathscr{F}\left(A_{M}, \mathfrak{g}\right) \subseteq \mathscr{F}^{1}\left(A_{M}, \mathfrak{g}\right) \cup(\Phi \otimes \mathrm{id}) \mathscr{F}\left(A_{N}, \mathfrak{g}\right)
$$

holds, then the inclusion

$$
\mathscr{R}_{1}^{1}\left(A_{M}, \theta\right) \subseteq \Pi\left(A_{M}, \theta\right) \cup(\Phi \otimes \mathrm{id}) \mathscr{R}_{1}^{1}\left(A_{N}, \theta\right)
$$

also holds. Pick $\omega \in \mathscr{R}_{1}^{1}\left(A_{M}, \theta\right)$. There are two cases to consider.

First suppose that $\omega \in \mathscr{F}^{1}\left(A_{M}, \mathfrak{g}\right)$. Using [28, Prop. 5.5] and induction on the dimension of $P^{1}$, we see that $\mathscr{R}_{1}^{1}\left(A_{M}\right) \subseteq\{0\} \cup \Phi\left(\mathscr{R}_{1}^{1}\left(A_{N}\right)\right)$. Hence, we may apply Lemma 8.3 (for $i=q=1$ ) to the one-element family $\left\{\Phi: A_{N} \hookrightarrow A_{M}\right\}$ and conclude that $\omega \in$ $\Pi\left(A_{M}, \theta\right) \cup(\Phi \otimes \mathrm{id}) \mathscr{R}_{1}^{1}\left(A_{N}, \theta\right)$, as required.

Finally, suppose that $\omega \notin \mathscr{F}^{1}\left(A_{M}, \mathfrak{g}\right)$. Then $\omega=\Phi \otimes \operatorname{id}\left(\omega^{\prime}\right)$, for some $\omega^{\prime} \in \mathscr{F}\left(A_{N}, \mathfrak{g}\right) \backslash$ $\mathscr{F}^{1}\left(A_{N}, \mathfrak{g}\right)$, by assumption (8.10). By Lemma 8.4, we have that $\omega^{\prime} \in \mathscr{R}_{1}^{1}\left(A_{N}, \theta\right)$. This verifies that (8.11) holds in this case, too, thereby completing the proof.

Theorem 8.5 improves on Theorem 1.5(2) from [28], where an extra assumption (injectivity of the transgression in degree 1) was required. Our stronger result here is of the same flavor as the equality (7.7) from Theorem 7.7, in the context provided by Remark 7.8. Namely, if $G$ is a $\mathbb{C}$-linear algebraic group with Lie algebra $\mathfrak{g}$ as above, and if $\rho: \pi_{1}(M) \rightarrow G$ is a representation near the origin 1 , then $\rho$ is either abelian or pulls back via $f$ from a $G$-representation of $\pi_{1}(N)$.

\section{QUASI-PROJECTIVE MANIFOLDS}

We conclude with another interesting class of examples where the uniform property holds for one-element families of maps, namely, regular maps between smooth, quasiprojective varieties. We also derive a non-compact analogue of Theorem 7.7 for a special class of quasi-projective manifolds, namely, complements of complex hyperplane arrangements. 
9.1. Mixed Hodge diagrams. Let $M$ be an irreducible, smooth, complex quasi-projective variety, or, for short, a quasi-projective manifold. Note that $M$ is a finite space. By resolution of singularities, we have that $M=\bar{M} \backslash D$, where $\bar{M}$ is a smooth projective variety, and $D \subset \bar{M}$ is a normal crossing divisor. A map between such pairs, $\bar{f}:(\bar{M}, D) \rightarrow$ $\left(\bar{M}^{\prime}, D^{\prime}\right)$, is called a regular morphism if the map $\bar{f}: \bar{M} \rightarrow \bar{M}^{\prime}$ is a regular map with the property that $\bar{f}^{-1}\left(D^{\prime}\right) \subseteq D$. Clearly, the restriction $f: \bar{M} \backslash D \rightarrow \bar{M}^{\prime} \backslash D^{\prime}$ is also a regular map. Conversely, any regular map between quasi-projective manifolds is induced by a regular morphism between convenient compactifications with normal crossing divisors.

We want to prove a quasi-projective analogue of Proposition 3.4. For that, we will need the theory of relative minimal models for mixed Hodge diagrams (MHDs, for short), developed by Cirici and Guillén in [5]. We start by recalling some pertinent definitions and results from [5].

The objects of the category FDGA are of the form $\left(A^{\bullet}, W_{\bullet}\right)$, where $\left(A^{\bullet}, d\right)$ is a CDGA defined over $\mathbb{Q}$ and $W$. is an increasing, multiplicative, regular, exhaustive filtration on $\left(A^{\bullet}, d\right)$, called a weight filtration. Such an object gives rise to a spectral sequence in the category of bigraded CDGAs, $\left\{E_{r}(A)\right\}_{r \geqslant 1}$, which converges to $H^{\bullet}(A)$. A morphism in FDGA is a CDGA map which respects filtrations. Such a morphism $\psi$ induces a map of spectral sequences, $\left\{E_{r}(\psi)\right\}_{r \geqslant 1}$.

The objects of the category MHD are strings of morphisms in FDGA defined over $\mathbb{C}$,

$$
\mathbf{H}: \quad A_{0} \stackrel{\psi_{0}}{\longrightarrow} A_{1} \longleftarrow \cdots \longrightarrow A_{\ell-1} \stackrel{\psi_{\ell-1}}{\longleftarrow} A_{\ell},
$$

where $\left(A_{0}, W\right)$ is defined over $\mathbb{Q}$ and all the induced maps $E_{2}\left(\psi_{i}\right)$ are isomorphisms. There are also additional data and axioms, related to the mixed Hodge structure (MHS) on $A_{\ell}$, see [5, Def. 3.1]. A morphism of mixed Hodge diagrams, $\Phi: \mathbf{H}^{\prime} \rightarrow \mathbf{H}$, is a tuple of FDGA maps, $\left(\Phi_{0}, \Phi_{1}, \ldots, \Phi_{\ell}\right)$, commuting with the maps $\psi^{\prime}$ and $\psi$, and such that $\Phi_{0}$ is defined over $\mathbb{Q}$. There is also an extra condition on $\Phi_{\ell}$ pertaining to the MHS, see [5, Def. 3.5].

9.2. The Gysin model of Morgan and Navarro. Returning to our setup, let $M$ be a quasi-projective manifold, and let $\bar{M}=M \cup D$ be a normal-crossing compactification. Given these data, Navarro constructs in [25] a mixed Hodge diagram $\mathbf{H}(\bar{M}, D)$, functorial with respect to regular morphisms of pairs (see also Hain [16]). Furthermore, there is an equivalence $A_{0}(\bar{M}, D) \simeq \Omega_{\mathbb{C}}(M)$ in CDGA, natural with respect to the pair $(\bar{M}, D)$. Moreover, $E_{1}\left(A_{0}(\bar{M}, D)\right)$ is isomorphic (as a bigraded CDGA) to $\operatorname{MG}(\bar{M}, D)$, the Gysin model of $M=\bar{M} \backslash D$ constructed by Morgan in [23, 24] (see also Dupont [11]). Note that this is a finite $\mathbb{C}$-model, defined over $\mathbb{Q}$, and that $\operatorname{MG}(\bar{M}, \varnothing)=\left(H^{\bullet}(\bar{M}), d=0\right)$.

Suppose $\bar{f}:(\bar{M}, D) \rightarrow\left(\overline{M^{\prime}}, D^{\prime}\right)$ is a regular morphism, such that the restriction $f: M \rightarrow$ $M^{\prime}$ preserves basepoints. Naturality in the sense of Navarro yields an equivalence

$$
\Omega_{\mathbb{C}}(f) \simeq \Phi_{0}(\bar{f})
$$


in $\mathbb{C}_{-A C D G A}$. Following Cirici and Guillén [5], we define

$$
\Phi(\bar{f})=E_{1}\left(\Phi_{0}(\bar{f})\right): \operatorname{MG}\left(\bar{M}^{\prime}, D^{\prime}\right) \rightarrow \operatorname{MG}(\bar{M}, D),
$$

over $\mathbb{C}$.

Proposition 9.1. Let $f: M \rightarrow M^{\prime}$ be a pointed, regular map between quasi-projective manifolds, inducing an injection on $H^{1}$. Extend $f$ to a regular morphism, $\bar{f}:(\bar{M}, D) \rightarrow$ $\left(\overline{M^{\prime}}, D^{\prime}\right)$, by adding divisors with normal crossings in a suitable manner. Then $\Omega_{\mathbb{C}}(f) \simeq$ $\Phi(\bar{f})$ in $\mathbb{C}_{-A C D G A}$.

Proof. Looking at $\mathbb{Q}$-components of MHDs and ignoring additional MHS data, we extract from [5, Theorems $3.17 \& 3.19$ ] the following commuting square in FDGA:

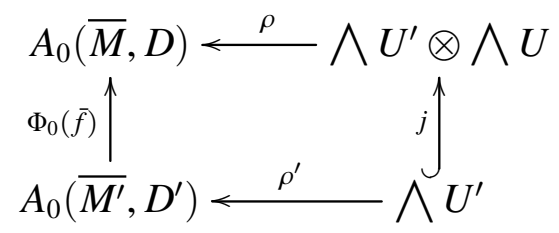

By [5, Lemma 3.4], the induced maps $E_{2}(\rho)$ and $E_{2}\left(\rho^{\prime}\right)$ are known to be isomorphisms. Hence, the maps $\rho$ and $\rho^{\prime}$ are quasi-isomorphisms. Furthermore, the CDGA diagram underlying (9.4) has the following properties: $\rho^{\prime}$ is a minimal model map, and $\rho$ is a relative minimal model map for $\Phi_{0}(\bar{f}) \circ \rho^{\prime}$, in the sense of $\S 3.3$.

Our injectivity assumption on $H^{1}(f)$, together with the equivalence from (9.2), imply that the map $\Phi_{0}(\bar{f}) \circ \rho^{\prime}$ is a 0 -equivalence. Using the discussion from $\S 3.3$, we infer that both $\bigwedge U^{\prime}$ and $\bigwedge U^{\prime} \otimes \wedge U$ are connected CDGAs. In particular, all maps from diagram (9.4) respect augmentations.

It's time now to take into account the available MHS data. We know from the work of Cirici and Guillén that the map $j$ is actually a morphism of mixed Hodge CDGAs, in the sense of [5, Definition 3.14]. According to Deligne's functorial splitting over $\mathbb{C}$ of mixed Hodge structures, we have the following identifications in CDGA,

$$
E_{1}\left(\bigwedge U^{\prime}\right)=\bigwedge U^{\prime}, \quad E_{1}\left(\bigwedge U^{\prime} \otimes \bigwedge U\right)=\bigwedge U^{\prime} \otimes \bigwedge U, \quad E_{1}(j)=j .
$$

This can be verified using the argument of Morgan from [23, Thm. 9.6]. See also [5, Lemma 3.20], where no extra finite-type assumptions are needed (over $\mathbb{C}$ ).

Applying the $E_{1}$ functor to diagram (9.4), we obtain the following commuting diagram in CDGA,

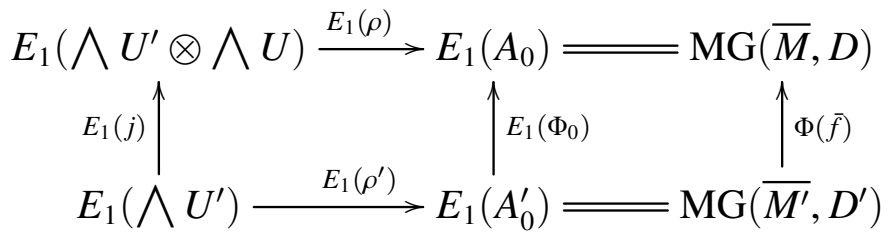


Here both horizontal arrows are quasi-isomorphisms, since $E_{2}(\rho)$ and $E_{2}\left(\rho^{\prime}\right)$ are isomorphisms. Since all CDGAs in sight are connected, (9.6) is a commuting diagram in ACDGA. The desired conclusion follows by putting together the information from displays (9.2) and (9.4)-(9.6).

Remark 9.2. As mentioned previously, it is known that the Navarro model $E_{1}\left(A_{0}(\bar{M}, D)\right)$ is isomorphic in CDGA to Morgan's Gysin model $\operatorname{MG}(\bar{M}, D)$. It is also known that the latter is functorial with respect to regular morphisms of pairs; see [11] for a convenient, explicit description of the CDGA map $\operatorname{MG}(\bar{f}): \operatorname{MG}\left(\bar{M}^{\prime}, D^{\prime}\right) \rightarrow \operatorname{MG}(\bar{M}, D)$ induced by $\bar{f}:(\bar{M}, D) \rightarrow\left(\overline{M^{\prime}}, D^{\prime}\right)$. But we do not know whether under this identification on objects the map $\operatorname{MG}(\bar{f})$ coincides with the map $\Phi(\bar{f})$ defined in (9.3). If that were the case, one could use [9, Ex. 5.3] to infer that the map $\Phi(\bar{f})=\operatorname{MG}(\bar{f})$ is injective, whenever $f: M \rightarrow$ $M^{\prime}$ is a regular surjection onto a curve, with connected generic fiber. This observation, together with Proposition 9.1, would then imply that the conclusions of Corollary 6.5 hold for regular admissible maps defined on quasi-projective manifolds, in the case when $q=1$.

9.3. Hyperplane arrangements. Let $\mathscr{A}$ be an arrangement of hyperplanes, that is, a finite, non-empty collection of complex affine hyperplanes in $\mathbb{C}^{\ell}$, for some $\ell>0$. The union of these hyperplanes is an affine hypersurface, $V_{\mathscr{A}}$, defined by an equation of the form $Q_{\mathscr{A}}=0$, where $Q_{\mathscr{A}}=\prod_{H \in \mathscr{A}} \alpha_{H}$ and $\alpha_{H}=0$ is a linear equation defining the hyperplane $H$. The complement of the arrangement, $M_{\mathscr{A}}=\mathbb{C}^{\ell} \backslash V_{\mathscr{A}}$, is a connected, smooth, quasi-projective variety, which has the homotopy type of a finite $\mathrm{CW}$-complex of dimension at most $\ell$.

A nice feature of this class of quasi-projective manifolds is that formality over $\mathbb{k}=\mathbb{R}$ or $\mathbb{C}$ holds in the following strong sense. For each $H \in \mathscr{A}$, the logarithmic 1-form

$$
\xi_{H}=\frac{1}{2 \pi \mathrm{i}} d \log \alpha_{H} \in \Omega_{\mathrm{dR}}\left(M_{\mathscr{A}}\right)
$$

is a closed form. Let $e_{H} \in H^{1}\left(M_{\mathscr{A}}, \mathbb{K}\right)$ be the cohomology class corresponding to $\left[\xi_{H}\right] \in$ $H_{\mathrm{dR}}^{1}\left(M_{\mathscr{A}}\right)$ under the de Rham isomorphism. It is known that $\left\{e_{H} \mid H \in \mathscr{A}\right\}$ forms a basis for $H^{1}\left(M_{\mathscr{A}}, \mathbb{k}\right)$. Thus, the $\mathbb{k}$-linear map $\xi_{\mathscr{A}}: H^{1}\left(M_{\mathscr{A}}, \mathbb{k}\right) \rightarrow \Omega_{\mathrm{dR}}^{1}\left(M_{\mathscr{A}}\right)$ sending each $e_{H}$ to $\xi_{H}$ yields an isomorphism $\left[\xi_{\mathscr{A}}\right]: H^{1}\left(M_{\mathscr{A}}, \mathbb{K}\right) \stackrel{\simeq}{\longrightarrow} H_{\mathrm{dR}}^{1}\left(M_{\mathscr{A}}\right)$.

The celebrated Brieskorn-Orlik-Solomon theorem (see [26]) states that the cohomology ring $H^{\bullet}\left(M_{\mathscr{A}}, \mathbb{Z}\right)$ is the quotient of the exterior algebra $\wedge^{\bullet} H^{1}\left(M_{\mathscr{A}}, \mathbb{Z}\right)$ by an ideal generated in degrees at least 2 and depending only on the intersection lattice of $\mathscr{A}$. Moreover, the extension of $\xi_{\mathscr{A}}$ to a CDGA map, $\xi_{\mathscr{A}}:\left(\bigwedge^{\bullet} H^{1}\left(M_{\mathscr{A}}, \mathbb{K}\right), d=0\right) \rightarrow \Omega_{\mathrm{dR}}^{\bullet}\left(M_{\mathscr{A}}\right)$, factors through a quasi-isomorphism

$$
\xi_{\mathscr{A}}:\left(H^{\bullet}\left(M_{\mathscr{A}}, \mathbb{k}\right), d=0\right) \longrightarrow \Omega_{\mathrm{dR}}^{\bullet}\left(M_{\mathscr{A}}\right) .
$$

We now suppose that the arrangement $\mathscr{A}$ is central, i.e., all hyperplanes $H \in \mathscr{A}$ pass through the origin $0 \in \mathbb{C}^{\ell}$. For the purpose of studying the fundamental group $\pi=$ 
$\pi_{1}\left(M_{\mathscr{A}}\right)$, we may assume that $\mathscr{A}$ is a central arrangement in $\mathbb{C}^{3}$. This can be achieved by taking a generic 3 -slice (if $\ell>3$ ), or taking the product with $\mathbb{C}^{3-\ell}$ (if $\ell<3$ ); neither operation changes the fundamental group of the complement.

Recall that $M_{\mathscr{A}}$ is a quasi-projective manifold. By the discussion from $\S 7.3$, there is a finite set $\mathscr{E}\left(M_{\mathscr{A}}\right)$ of admissible maps $f: M_{\mathscr{A}} \rightarrow M_{f}$ (up to reparametrization at the target), such that $M_{f}$ is a smooth curve with $\chi\left(M_{f}\right)<0$. It turns out that the mixed Hodge structure on $M_{\mathscr{A}}$ is pure of weight 2. Consequently, each curve $M_{f}$ must be of the form $\mathbb{C P}^{1} \backslash\{k$ points $\}$, for some $k \geqslant 3$.

Falk and Yuzvinsky gave in [12] a particularly nice, combinatorial description of the set $\mathscr{E}\left(M_{\mathscr{A}}\right)$, well-suited for our purposes here (see also [8, §5] and [27, §6]). The key combinatorial notion is that of a multinet. Given an integer $k \geqslant 3$, a $k$-multinet $\mathcal{N}$ on a central arrangement $\mathscr{A}$ in $\mathbb{C}^{3}$ consists of a partition, $\mathscr{A}=\mathscr{A}_{1} \sqcup \cdots \sqcup \mathscr{A}_{k}$, and a multiplicity function, $m: \mathscr{A} \rightarrow \mathbb{N}$, satisfying several axioms, one of which being that the sum $\sum_{H \in \mathscr{A}_{i}} m_{H}$ is independent of $i \in[k]$.

The multinet axioms imply that the polynomials $Q_{i}=\prod_{H \in \mathscr{A}_{i}} \alpha_{H}^{m_{H}}$ belong to a pencil of curves, that is, for each $i>2$ there are constants $a_{i}$ and $b_{i}$ such that $Q_{i}=a_{i} Q_{1}+b_{i} Q_{2}$. Consider the central line arrangement $\mathscr{L}=\left\{\mathscr{L}_{1}, \ldots, \mathscr{L}_{k}\right\}$ in $\mathbb{C}^{2}$, with $\mathscr{L}_{i}=\left\{g_{i}=0\right\}$, where $g_{1}=z_{1}, g_{2}=z_{2}$, and $g_{i}=a_{i} z_{1}+b_{i} z_{2}$ for $i>2$. Let $f_{\mathcal{N}}: M_{\mathscr{A}} \rightarrow M_{\mathscr{L}}$ be the regular map with components $\left(Q_{1}, Q_{2}\right)$. Projectivizing, we obtain an admissible map,

$$
f_{\mathcal{N}}: M_{\mathscr{A}} \rightarrow \mathbb{C P}^{1} \backslash\{k \text { points }\}
$$

More generally, there is a complete set of representatives for $\mathscr{E}\left(M_{\mathscr{A}}\right)$ consisting of admissible maps $f_{\mathcal{N}}: M_{\mathscr{A}} \rightarrow \mathbb{C P}^{1} \backslash\{k$ points $\}$ obtained by restricting to $M_{\mathscr{A}}$ the map $f_{\mathcal{N}}: M_{\mathcal{B}} \rightarrow \mathbb{C P}^{1} \backslash\{k$ points $\}$, where $\mathcal{N}$ is a $k$-multinet on a sub-arrangement $\mathcal{B} \subseteq \mathscr{A}$; see [27, Corollary 6.6].

Now set $n=|\mathscr{A}|$, and identify $\pi_{\text {abf }}=\mathbb{Z}^{n}$. Let $\mathscr{C}$ be the Boolean arrangement in $\mathbb{C}^{n}$, consisting of all coordinate hyperplanes. Clearly, $M_{\mathscr{C}}=\left(\mathbb{C}^{\times}\right)^{n}$. Consider the regular map $f_{0}: M_{\mathscr{A}} \rightarrow M_{\mathscr{C}}$ with components $\left(\alpha_{H}\right)_{H \in \mathscr{A}}$. As noted for instance in [8, Lem. 5.1], the induced homomorphism, $\left(f_{0}\right)_{\sharp}: \pi_{1}\left(M_{\mathscr{A}}\right) \rightarrow \pi_{1}\left(M_{\mathscr{C}}\right)$, coincides with the canonical projection, abf : $\pi \rightarrow \pi_{\text {abf }}$. We let $E\left(M_{\mathscr{A}}\right)=\mathscr{E}\left(M_{\mathscr{A}}\right) \cup\left\{f_{0}\right\}$, as in (7.2).

By construction, all maps $f \in E\left(M_{\mathscr{A}}\right)$ are of the form $f: M \rightarrow M_{f}$, where $M=M_{\mathscr{A}}$ and $M_{f}=M_{\mathscr{A}_{f}}$, for some (affine) arrangement $\mathscr{A}_{f}$.

Proposition 9.3. Let $\mathscr{A}$ be a central hyperplane arrangement in $\mathbb{C}^{3}$, and fix a basepoint in $M_{\mathscr{A}}$. For $\mathbb{K}=\mathbb{R}$ or $\mathbb{C}$, we have that $\Omega_{\mathbb{k}}(f) \simeq H^{\bullet}(f, \mathbb{K})$ in $\mathbb{R}-\mathrm{ACDGA}_{0}$, uniformly with respect to $f \in E\left(M_{\mathscr{A}}\right)$. 
Proof. In view of the Brieskorn-Orlik-Solomon isomorphism (9.8), it is enough to check the commutativity of the following diagram in CDGA:

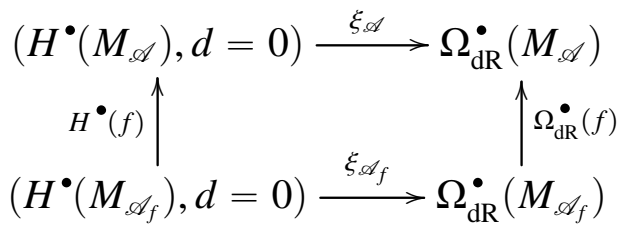

Since the cohomology ring of an arrangement complement is generated in degree 1, we may assume that $\cdot=1$ in the above diagram. Using the explicit construction of the map $\xi$ in degree 1 , we can further reduce to showing that $\Omega_{\mathrm{dR}}(f)\left(d \log \alpha_{H^{\prime}}\right)$ belongs to the $\mathbb{Z}$-span of $\left\{d \log \alpha_{H} \mid H \in \mathscr{A}\right\}$, for every $H^{\prime} \in \mathscr{A}_{f}$.

First assume $f=f_{0}$. Then the claim follows from the formula $\Omega_{\mathrm{dR}}\left(f_{0}\right)\left(d \log z_{H}\right)=$ $d \log \alpha_{H}$, for every $H \in \mathscr{A}$, which in turn follows directly from the definition of $f_{0}$.

Next assume $f=f_{\mathcal{N}}$, for some multinet $\mathcal{N}$ on a sub-arrangement $\mathcal{B} \subseteq \mathscr{A}$. Clearly, we may assume that $\mathcal{B}=\mathscr{A}$. The claim is now an easy consequence of the formula $\Omega_{\mathrm{dR}}\left(f_{\mathcal{N}}\right)\left(d \log g_{i}\right)=\sum_{H \in \mathscr{A}_{i}} m_{H} d \log \alpha_{H}$, which is verified in [27, Lem. 6.3].

Theorem 9.4. Let $\mathscr{A}$ be a central hyperplane arrangement with complement $M=M_{\mathscr{A}}$. Write $\pi=\pi_{1}(M)$, and, for each map $f: M \rightarrow M_{f}$ in $E(M)$, set $\pi_{f}=\pi_{1}\left(M_{f}\right)$. Let $G$ be a $\mathbb{C}$-linear algebraic group with non-abelian Lie algebra $\mathfrak{g} \subseteq \mathfrak{s l}_{2}(\mathbb{C})$, and let $\iota: G \rightarrow \mathrm{GL}(V)$ be a rational representation. Then,

$$
\operatorname{Hom}(\pi, G)_{(1)}=\bigcup_{f \in E(M)} f_{\sharp}^{!} \operatorname{Hom}\left(\pi_{f}, G\right)_{(1)},
$$

and, for $i=r=1$ or $i=0$ and $r \geqslant 1$,

$$
\mathscr{V}_{r}^{i}(\pi, \iota)_{(1)}=\bigcup_{f \in E(M)} f_{\sharp}^{!} \mathscr{V}_{r}^{i}\left(\pi_{f}, \iota\right)_{(1)} .
$$

Proof. As noted before, we may assume $\ell=3$. The argument we give is closely modeled on the proof of Theorem 7.7. To begin with, note that the conclusions of Lemma 7.6 hold for the formal, quasi-projective manifold $M=M_{\mathscr{A}}$, with the same proof. Next, consider the map $f_{0}: M_{\mathscr{A}} \rightarrow M_{\mathscr{C}}$, and the induced CDGA map $H^{\bullet}\left(f_{0}\right): H^{\bullet}\left(M_{\mathscr{C}}\right) \rightarrow H^{\bullet}\left(M_{\mathscr{A}}\right)$, where both differentials are 0 . Since $M_{\mathscr{C}}=\left(\mathbb{C}^{\times}\right)^{n}$, where $n=|\mathscr{A}|$, we may identify $H^{\bullet}\left(M_{\mathscr{C}}\right)$ with $\wedge^{\bullet} H^{1}\left(M_{\mathscr{C}}\right)$. Furthermore, $H^{1}\left(f_{0}\right)$ is an isomorphism, by construction. Hence, Lemma 7.3 may be applied to the map $\Phi=H^{\bullet}\left(f_{0}\right)$.

By Proposition 9.3, we have that $\Omega(f) \simeq H^{\bullet}(f)$ in ACDGA $_{0}$, uniformly with respect to $f \in E\left(M_{\mathscr{A}}\right)$. We may now apply Theorem 6.4 for $q=1$ to the family of pointed continuous maps $\left\{f: M_{\mathscr{A}} \rightarrow M_{\mathscr{A}_{f}}\right\}_{f \in E\left(M_{\mathscr{A}}\right)}$ and the family of CDGA maps $\left\{H^{\bullet}(f): H^{\bullet}\left(M_{\mathscr{A}_{f}}\right) \rightarrow\right.$ $\left.H^{\bullet}\left(M_{\mathscr{A}}\right)\right\}_{f \in E\left(M_{\mathscr{A}}\right)}$, where again all differentials are 0 . The rest of the argument goes exactly as in the proof of Theorem 7.7. 


\section{ACKNOWLEDGEMENT}

Part of this work was done while the second author visited the Institute of Mathematics of the Romanian Academy in June, 2016. He thanks IMAR for its hospitality, support, and excellent research atmosphere.

\section{REFERENCES}

[1] A. Adem, F.R. Cohen, Commuting elements and spaces of homomorphisms, Math. Ann. 338 (2007), no. 3, 587-626. MR2317932 4.5

[2] D. Arapura, Geometry of cohomology support loci for local systems I, J. Algebraic Geom. 6 (1997), no. 3, 563-597. MR1487227 1.3, 7.3

[3] A. Borel, R. Friedman, J.W. Morgan, Almost commuting elements in compact Lie groups, Mem. Amer. Math. Soc. 157 (2002), no. 747. MR1895253 4.5

[4] N. Budur, B. Wang, Cohomology jump loci of differential graded Lie algebras, Compos. Math. 151 (2015), no. 8, 1499-1528. MR3383165 1.1, 5.1, 5.2

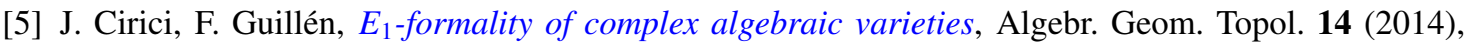
no. 5, 3049-3079. MR3276854 1.4, 9.1, 9.1, 9.2, 9.2, 9.2, 9.2

[6] K. Corlette, C. Simpson, On the classification of rank-two representations of quasiprojective fundamental groups, Compos. Math. 144 (2008), no. 5, 1271-1331. MR2457528 1.3, 7.8

[7] P. Deligne, P. Griffiths, J.W. Morgan, D. Sullivan, Real homotopy theory of Kähler manifolds, Invent. Math. 29 (1975), no. 3, 245-274. MR0382702 1.3, 3.5, 7.2

[8] G. Denham, A.I. Suciu, Multinets, parallel connections, and Milnor fibrations of arrangements, Proc. London Math. Soc. 108 (2014), no. 6, 1435-1470. MR3218315 9.3, 9.3

[9] A. Dimca, S. Papadima, Non-abelian cohomology jump loci from an analytic viewpoint, Commun. Contemp. Math. 16 (2014), no. 4, 1350025, 47 pp. MR3231055 1.1, 3.2, 4.6, 4.6, 4.6, 4.6, 5.1, 5.1, 5.2, 5.2, 6.1, 6.1, 6.1, 7.4, 7.8, 7.5, 8.1, 9.2

[10] A. Dimca, S. Papadima, A.I. Suciu, Topology and geometry of cohomology jump loci, Duke Math. Journal 148 (2009), no. 3, 405-457. MR2527322 1.3, 7.4

[11] C. Dupont, The Orlik-Solomon model for hypersurface arrangements, Ann. Inst. Fourier (Grenoble), 65 (2015), no. 6, 2507-2545. MR3449588 9.2, 9.2

[12] M. Falk, S. Yuzvinsky, Multinets, resonance varieties, and pencils of plane curves, Compositio Math. 143 (2007), no. 4, 1069-1088. MR2339840 1.3, 9.3

[13] Y. Félix, S. Halperin, J.-C. Thomas, Rational homotopy theory, Grad. Texts in Math., vol. 205, Springer-Verlag, New York, 2001. MR1802847 3.2, 3.3, 7.2, 8.2, 8.2

[14] W. Goldman, Topological components of spaces of representations, Invent. Math. 93 (1988), no. 3, 557-607. MR0952283 4.5

[15] W. Goldman, J. Millson, The deformation theory of representations of fundamental groups of compact Kähler manifolds, Inst. Hautes Études Sci. Publ. Math. 67 (1988), 43-96. MR0972343 4, 4.1, 4.6, 4.6, 6.1

[16] R. Hain, The de Rham homotopy theory of complex algebraic varieties. I. K-Theory 1 (1987), no. 3, 271-324. MR0908993 9.2

[17] S. Halperin, Lectures on minimal models, Mém. Soc. Math. France, Sér. 2, 9-10 (1983), 1-261. MR0736299 3.2, 3.3

[18] M. Kapovich, J. Millson, On representation varieties of Artin groups, projective arrangements and the fundamental groups of smooth complex algebraic varieties, Inst. Hautes Études Sci. Publ. Math. 88 (1998), 5-95. MR1733326 1.1 
[19] D. Lehmann, Théorie homotopique des formes diférentielles (d'après D. Sullivan), Astérisque, no. 45, Société Mathématique de France, Paris, 1977. MR0488041 3.2

[20] F. Loray, J.V. Pereira, F. Touzet, Representations of quasiprojective groups, flat connections and transversely projective foliations, J. Éc. Polytech. Math. 3 (2016), 263-308. MR3522824 1.3, 7.8

[21] M. Manetti, Deformation theory via differential graded Lie algebras, arxiv:math. AG/0507284v1. 4, 4.2

[22] A. Măcinic, S. Papadima, R. Popescu, A.I. Suciu, Flat connections and resonance varieties: from rank one to higher ranks, Trans. Amer. Math. Soc. 369 (2017), no. 2, 1309-1343. MR3572275 1.3, 5.4, 5.4, 5.5, 7.1, 7.1, 7.4, 7.5, 7.10, 8.3, 8.3, 8.3

[23] J.W. Morgan, The algebraic topology of smooth algebraic varieties, Inst. Hautes Études Sci. Publ. Math. 48 (1978), 137-204. MR0516917 1.4, 3.2, 9.2, 9.2

[24] J.W. Morgan, Correction to: "The algebraic topology of smooth algebraic varieties", Inst. Hautes Études Sci. Publ. Math. 64 (1986), 185. MR0876163 9.2

[25] V. Navarro Aznar, Sur la théorie de Hodge-Deligne, Invent. Math. 90 (1987), no. 1, 11-76. MR0906579 9.2

[26] P. Orlik, H. Terao, Arrangements of hyperplanes, Grundlehren Math. Wiss., vol. 300, Springer-Verlag, Berlin, 1992. MR12174889.3

[27] S. Papadima, A.I. Suciu, The Milnor fibration of a hyperplane arrangement: from modular resonance to algebraic monodromy, Proc. London Math. Soc. 114 (2017), no. 6, 961-1004. MR3661343 9.3, 9.3, 9.3

[28] S. Papadima, A.I. Suciu, The topology of compact Lie group actions through the lens of finite models, Int. Math. Res. Notices (2018), 1-47, doi:10.1093/imrn/rnx294. 1.3, 8.3, 8.3

[29] S. Papadima, A.I. Suciu, Rank two topological and infinitesimal embedded jump loci of quasiprojective manifolds, J. Inst. Math. Jussieu (2018), 1-35, doi:10.1017/S1474748018000063. 1.3

[30] D. Quillen, Rational homotopy theory, Ann. of Math. (2) 90 (1969), 205-295. MR0258031 3

[31] A.S. Rapinchuk, V.V. Benyash-Krivetz, V.I. Chernousov, Representation varieties of the fundamental groups of compact orientable surfaces, Israel J. Math. 93 (1996), no. 1, 29-71. MR1380633 4.5

[32] D. Sullivan, Infinitesimal computations in topology, Inst. Hautes Études Sci. Publ. Math. (1977), no. 47, 269-331. MR0646078 3, 1.4, 3, 3.2, 3.5

[33] J.-Cl. Tougeron, Idéaux de fonctions différentiables, Ergeb. Math. Grenzgeb., vol. 71, Springer-Verlag, Berlin-New York, 1972. MR0440598 2, 2, 6.2

[34] M. Vigué-Poirrier, Réalisation de morphismes donnés en cohomologie et suite spectrale d'EilenbergMoore, Trans. Amer. Math. Soc. 265 (1981), no. 2, 447-484. MR0610959 1.4, 3.5, 3.5

[35] G.W. Whitehead, Elements of homotopy theory, Grad. Texts in Math., vol. 61, Springer-Verlag, New York-Berlin, 1978. MR0516508 5.1, 5.5

Simion Stoilow Institute of Mathematics, P.O. Box 1-764, RO-014700 Bucharest, Romania

E-mail address: Stefan.Papadima@imar.ro

Department of Mathematics, Northeastern University, Boston, MA 02115, USA

E-mail address: a. suciu@northeastern. edu

$U R L$ : web. northeastern . edu/suciu/ 